\title{
Inhibition of DNMT1 and ERRa crosstalk suppresses breast cancer via derepression of IRF4
}

\author{
Mathieu Vernier (i) ${ }^{1}$. Shawn McGuirk ${ }^{1}$ - Catherine R. Dufour ${ }^{1}$ - Liangxinyi Wan ${ }^{1}$ - Etienne Audet-Walsh $\mathbb{D}^{1,3}$. \\ Julie St-Pierre ${ }^{1,2,4} \cdot$ Vincent Giguère $\mathbb{I}^{1,2}$
}

Received: 8 May 2020 / Revised: 10 August 2020 / Accepted: 17 August 2020 / Published online: 27 August 2020

(c) The Author(s) 2020. This article is published with open access

\begin{abstract}
DNA methylation is implicated in the acquisition of malignant phenotypes, and the use of epigenetic modulating drugs is a promising anti-cancer therapeutic strategy. 5-aza-2'deoxycytidine (decitabine, 5-azadC) is an FDA-approved DNA methyltransferase (DNMT) inhibitor with proven effectiveness against hematological malignancies and more recently triplenegative breast cancer (BC). Herein, genetic or pharmacological studies uncovered a hitherto unknown feedforward molecular link between DNMT1 and the estrogen related receptor $\alpha(E R R \alpha)$, a key transcriptional regulator of cellular metabolism. Mechanistically, DNMT1 promotes ERR $\alpha$ stability which in turn couples DNMT1 transcription with that of the methionine cycle and S-adenosylmethionine synthesis to drive DNA methylation. In vitro and in vivo investigation using a pre-clinical mouse model of $\mathrm{BC}$ demonstrated a clear therapeutic advantage for combined administration of the ERR $\alpha$ inhibitor $\mathrm{C} 29$ with 5-azadC. A large-scale bisulfite genomic sequencing analysis revealed specific methylation perturbations fostering the discovery that reversal of promoter hypermethylation and consequently derepression of the tumor suppressor gene, IRF4, is a factor underlying the observed BC suppressive effects. This work thus uncovers a critical role of ERR $\alpha$ in the crosstalk between transcriptional control of metabolism and epigenetics and illustrates the potential for targeting ERR $\alpha$ in combination with DNMT inhibitors for BC treatment and other epigenetics-driven malignancies.
\end{abstract}

Supplementary information The online version of this article (https:// doi.org/10.1038/s41388-020-01438-1) contains supplementary material, which is available to authorized users.

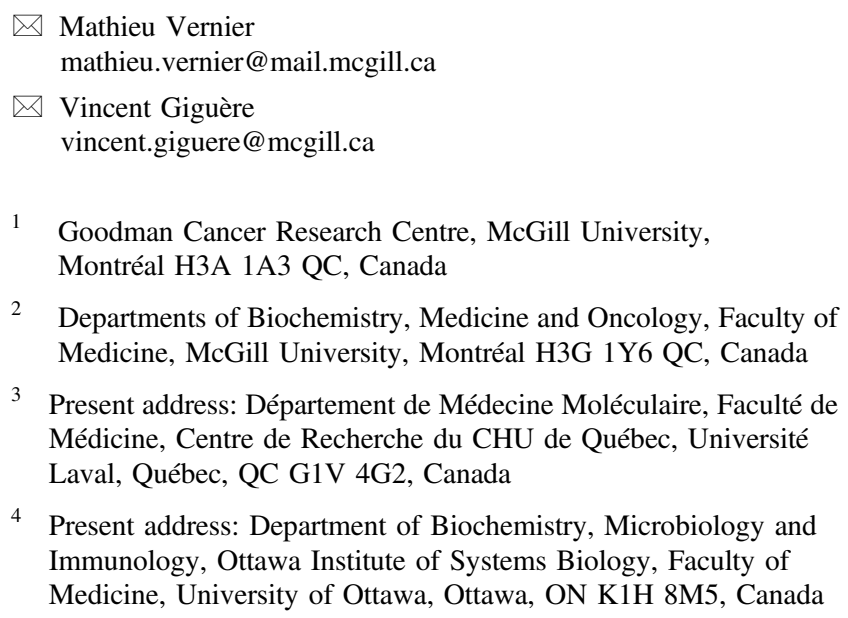

2 Departments of Biochemistry, Medicine and Oncology, Faculty of Medicine, McGill University, Montréal H3G 1Y6 QC, Canada

3 Present address: Département de Médecine Moléculaire, Faculté de Médicine, Centre de Recherche du CHU de Québec, Université Laval, Québec, QC G1V 4G2, Canada

4 Present address: Department of Biochemistry, Microbiology and Immunology, Ottawa Institute of Systems Biology, Faculty of Medicine, University of Ottawa, Ottawa, ON K1H 8M5, Canada

\section{Introduction}

Methylation of DNA is an evolutionarily conserved mechanism that allows control of gene expression by modulating chromatin accessibility to specific transcription factors (TFs) [1, 2]. This epigenetic process is crucial for proper mammalian development, essential for cellular differentiation and plays a determinant role in maintaining genomic stability [3-5]. Aberrant DNA methylation patterns have been observed in a large variety of diseases including obesity, diabetes, neurodegeneration, and cancer, thus prompting intense research to fully discern its regulatory modes and fuel the development of new therapeutic approaches [6-8].

In mammals, DNA methylation occurs at the fifth position of cytosine to produce 5-methylcytosine (5-mC). Maintenance of genomic methylation is ensured by the DNA methyltransferase DNMT1 which copies the DNA methylation pattern from the parental DNA strand onto the newly synthesized daughter strand during replication [9]. DNA methylation is also responsive and adaptive to environmental changes by modulating the expression of genes [10]. Two 
other methyltransferases, DNMT3A and 3B, have been described to methylate DNA de novo. Conversely, the ten-eleven translocation (TET) enzymes 1, 2, and 3 have the capacity to demethylate the genome [11-13].

The methyl group needed for DNA methylation is typically derived from dietary methionine, which is metabolized through the methionine cycle upon entering the cell. In this pathway, a molecule of ATP is transferred to methionine for S-adenosylmethionine (SAM) synthesis, the primary methyl donor for most biological methylation reactions [14]. After the methyl group is donated, the resulting S-adenosylhomocysteine (SAH) is either recycled or metabolized into cysteine, a nonessential amino acid that serves as a key building block for glutathione synthesis. The demethylation process catalyzed by the TET enzymes is also dependent on metabolite levels, and requires $\alpha$-ketoglutarate $(\alpha \mathrm{KG})$, which can be generated from the tricarboxylic acid (TCA) cycle and glutaminolysis [12]. While DNA methylation and demethylation are clearly intrinsically linked to metabolism, the mechanisms that coordinate these programs remain poorly understood.

In the context of breast cancer (BC), several aspects of cellular metabolism are controlled by members of the estrogen related receptor (ERR) family [15]. The ERRs are orphan nuclear receptors that regulate a large variety of metabolic gene networks implicated in glycolysis, glutaminolysis, mitochondrial biogenesis, and cellular respiration $[16,17]$. Recently, we have shown that ERR $\alpha$ is also a transcriptional regulator of the folate cycle, a metabolic pathway closely related to the methionine cycle [18]. ERR $\alpha$ also plays a central role in regulating the adaptive metabolic processes used by breast tumors to thrive in conditions of fluctuating nutrient availability [19]. Furthermore, high ERR $\alpha$ transcript levels are associated with the HER2 positive and triple-negative (TN) molecular subtypes known to be among the most aggressive forms of the disease [20].

In this study, we identify ERR $\alpha$ as a direct link between cellular metabolism and DNA methylation. We first show that inhibition of ERR $\alpha$ activity diminishes the expression of methionine cycle enzymes and markedly reduces DNMT1 transcription resulting in a global loss of cellular DNA methylation. In a feedforward regulatory loop, DNMT1 elevated ERR $\alpha$ protein, and levels of DNMT1 mRNA correlated with high ERR $\alpha$ activity in BC patients. Importantly, pharmacological inhibition of ERR $\alpha$ further sensitized BC cells in vitro and in vivo to the anti-neoplastic effects of the DNMT inhibitor 5-aza-2'-deoxycytidine (decitabine, 5AzadC). The clinical significance of our findings is further supported by genome-wide bisulfite sequencing, revealing that co-administration of ERR $\alpha$ and DNMT1 inhibitors leads to promoter demethylation and re-expression of IRF4encoding Interferon Regulatory Factor- 4 and found herein to exhibit tumor-suppressor activity in BC cells.

\section{Results}

\section{ERRa regulates the expression of enzymes of the methionine cycle and DNA methylation}

DNA methylation is dependent on cellular metabolic activity, specifically the methionine cycle (Fig. 1a), and considering that the ERRs are key transcriptional regulators of cell metabolism, we investigated whether ERR $\alpha$ is directly implicated in this process. To this end, we interrogated ERR $\alpha$ ChIP-seq datasets obtained in the BC cell lines BT474 and SKBR3 [21]. These cells are characterized by high expression of the receptor tyrosine kinase HER2, known to drive ERR $\alpha$ function [22, 23]. Consequently, BT474 and SKBR3 cells possess high ERR $\alpha$ activity and represent ideal models for our investigation. ERR $\alpha$-binding sites were found near the transcriptional start sites of the DNA methytransferase genes DNMT1 and DNMT3A, the DNA demethylation enzyme genes TET2, TET3, and TDG, and the $A H C Y$ gene of the methionine cycle in both cell lines (Supplementary Fig. 1a). ERR $\alpha$ was found specifically bound to regulatory regions near MATIA in BT474 cells and MAT2A in SKBR3 cells suggesting cell-specific differences in isoform expression (Supplementary Fig. 1a). ChIP-qPCR validated the recruitment of ERR $\alpha$ to these sites which was lost when cells were treated with the specific ERR $\alpha$ inhibitor C29 [24] (Fig. 1b). ERR $\alpha$ inhibition with $\mathrm{C} 29$ led to a significant induction of the DNAdemethylating genes TET3 and TDG along with an observed inverse regulation of DNA methylating genes with downregulation of DNMT1 and upregulation of DNMT3A (Fig. 1c, d). Further, targeting ERR $\alpha$ diminished the expression of $A H C Y$, as well as that of MATIA and MAT2A, respectively, in BT474 and SKBR3 cells (Fig. 1c, d), the latter in line with ERR $\alpha$ ChIP-seq binding profiles. Immunoblot analysis confirmed similar effects on the protein levels of these genes following ERR $\alpha$ knockdown or inhibition by $\mathrm{C} 29$ in both HER2 + cell lines (Fig. 1e-h). Although, HER2 can positively regulate ERR $\alpha$ activity, ERR $\alpha$ is also expressed in the ER+ and TN BC subtypes. To verify whether ERR $\alpha$ regulation of DNA methylating enzyme expression is subtype-specific, we looked at protein levels of DNMT1 after knockdown of ERR $\alpha$ by RNA interference in MCF7 cells, an ER+ BC cell line, as well as in the three TNBC cell lines MDA-MB-231, MDA-MB-436, and MDA-MB-468. In each case, impairment of ERR $\alpha$ function reduced DNMT1 protein levels (Supplementary Fig. 1b-e).

Importantly, this specific involvement of ERR $\alpha$ in the regulation of DNA methylation is not restricted to human cancer cells. Drug-induced inhibition of ERR $\alpha$ by C29 in the mouse cell lines NIC-5231 and NIC-5257, derived from ErbB2-driven mammary tumors [25], also led to a stark reduction in DNMT1 protein (Fig. 1i, j). This mechanism is 
A

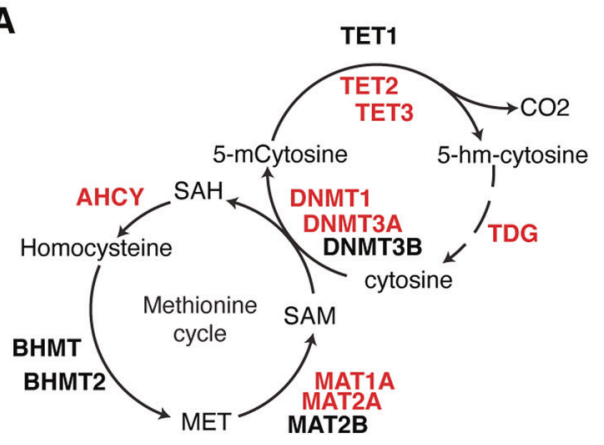

C

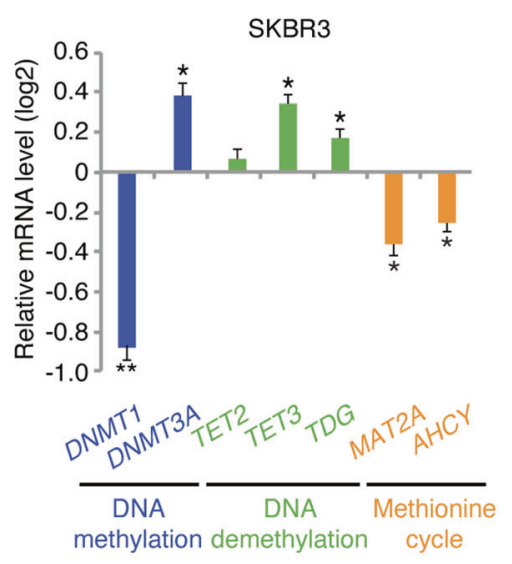

E

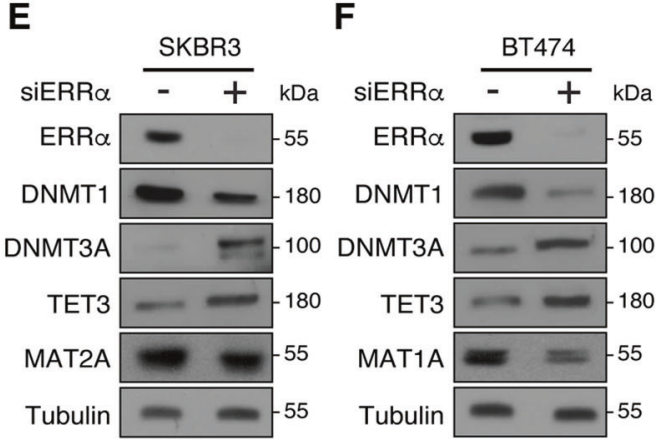

I

J

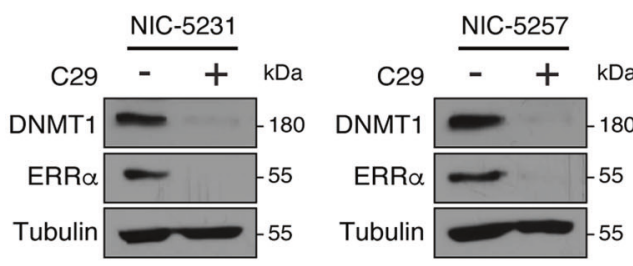

B

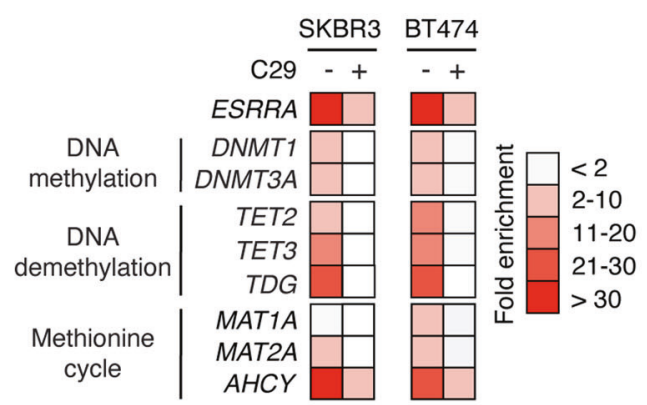

D

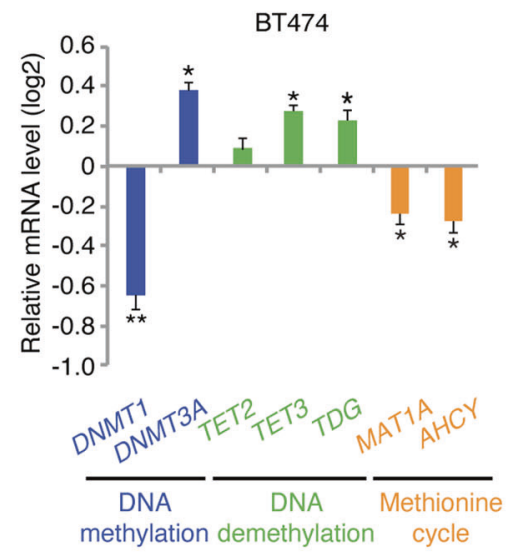

G

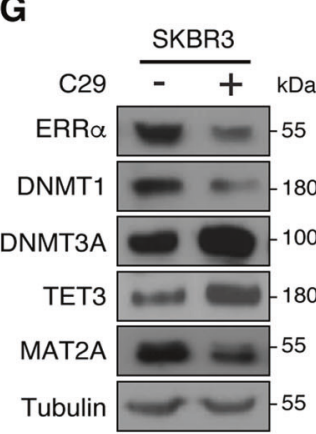

H

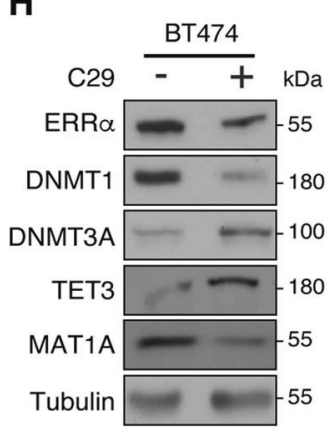

$\mathbf{K}$

$\mathbf{L}$

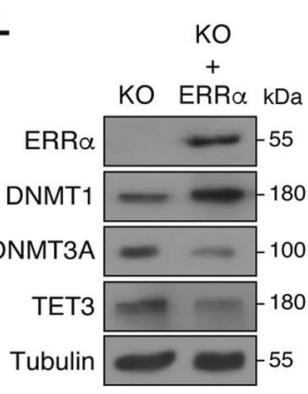

also conserved in normal cells whereby ERR $\alpha$ knockout mouse embryonic fibroblasts (ERR $\alpha$ KO MEFs) exhibited similar alterations in DNMT isoforms and TET expression as compared to $\mathrm{BC}$ cells, a phenotype reversed by ectopic expression of ERR $\alpha$ (Fig. 1k, 1).

\section{ERRa controls DNA methylation}

To investigate the influence of ERR $\alpha$ on the methionine cycle and DNA methylation programs, we measured the steady-state levels of methionine cycle intermediates in 
Fig. 1 ERR $\alpha$ regulates genes involved in DNA methylation and the methionine cycle. a Schematic representation of the relationship between the methionine cycle and DNA methylation. Enzymes in red were identified as direct ERR $\alpha$ target genes by ChIP-seq. b ChIPqPCR analysis of ERR $\alpha$ binding on the target genes identified in (a) following treatment with $\mathrm{C} 29$ for $24 \mathrm{~h}(n=3)$. c, $\mathbf{d}$ qRT-PCR analysis of SKBR3 (c) and BT474 (d) cells after treatment with C29 for $24 \mathrm{~h}$. Control cells treated with vehicle were set at 0 . Results reflect three independent experiments performed in triplicate. e, f Immunoblot analysis of SKBR3 (e) and BT474 (f) cells post-transfection for $48 \mathrm{~h}$ with siRNAs against ERR $\alpha$. Tubulin is shown as a loading control. $\mathbf{g}, \mathbf{h}$ Immunoblot analysis of SKBR3 (g) and BT474 (h) cells following treatment with $\mathrm{C} 29$ for $24 \mathrm{~h}$. Tubulin is shown as a loading control. $\mathbf{i}, \mathbf{j}$ Immunoblots of ERR $\alpha$ and DNMT1 in the mouse BC cell lines NIC-5231 (i) and NIC-5257 (j) after treatment with C29 for $24 \mathrm{~h}$. Tubulin is shown as a loading control. $\mathbf{k}$ Immunoblots of ERR $\alpha$ KO MEFs compared to WT. Tubulin is shown as a loading control. 1 Immunoblots of ERR $\alpha$ KO MEFs with ectopic expression of ERR $\alpha$ compared to the parental KO MEFs. Tubulin is shown as a loading control. Data shown in $\mathbf{c}$ and $\mathbf{d}$ represent means \pm SEM. $* p<0.05$, ** $p<0.01$; Student's $t$ test.

SKBR3 cells after pharmacological inhibition of ERR $\alpha$ with $\mathrm{C} 29$ for $24 \mathrm{~h}$. Impeding $\mathrm{ERR} \alpha$ resulted in a significant accumulation of all intermediates of this metabolic process (Fig. 2a). Moreover, C29-mediated ERR $\alpha$ inhibition led to a marked decrease in global DNA methylation in both SKBR3 and BT474 cell lines, exemplified by a significant reduction in total 5-methylcytosine levels (Fig. 2b, c). Importantly, ERR $\alpha \mathrm{KO}$ MEFs also displayed a decreased level of DNA methylation compared to WT MEFs (Supplementary Fig. 2).

The accumulation of methionine cycle intermediates might be the consequence of a reduced metabolic rate due to the decreased expression of the methionine cycle enzymes we observed (Fig. 1c-h) or a bottleneck downstream in the transfer of a methyl group from SAM to DNA. To measure the metabolic rate of the methionine cycle, we designed an isotope tracer experiment whereby SKBR3 cells were first treated with $\mathrm{C} 29$ for $24 \mathrm{~h}$, followed by incubation with labeled methionine $\left({ }^{13} \mathrm{C}_{5}{ }^{15} \mathrm{~N}\right.$-methionine) for $2 \mathrm{~h}$. Given that methionine is an essential amino acid, we could follow the incorporation of labeled atoms from dietary methionine into SAM, SAH, and homocysteine by liquid chromatography coupled to mass spectrometry (LC/MS). Interestingly, while we could not detect homocysteine in this setting, C29treated cells displayed an increase of labeled methionine (Fig. 2d). Of note, C29 had no significant impact on SAM and SAH levels, suggesting a similar rate of the methionine cycle compared to control (Fig. 2d).

We then quantified the levels of labeled 5methylcytosine arising from labeled methionine. BC cells were first treated with $\mathrm{C} 29$ for $24 \mathrm{~h}$ and then incubated with labeled methionine for another $24 \mathrm{~h}$ to allow for labeled methyl incorporation prior to genomic DNA isolation. As expected, diminished levels of labeled 5-methylcytosine were observed when $\operatorname{ERR} \alpha$ activity was impaired (Fig. 2e, f). Notably, this effect was largely rescued by exogenous expression of DNMT1 (Fig. 2g, h), highlighting that DNMT1 is the rate-limiting enzyme and is critical for driving DNA methylation in this context.

\section{ERRa activity correlates with DNMT1 expression in $B C$ patients}

We next re-analyzed publicly available gene expression datasets from cohorts of BC patient tumors of mixed molecular subtypes to determine whether ERR $\alpha$ activity correlates with the expression of DNA methylation regulators. For this, we utilized a previously established 121gene $\mathrm{ERR} \alpha$ signature shown to cluster BC patients into groups of low or high $\mathrm{ERR} \alpha$ activity independent of their BC molecular subtype [23]. To this gene list, we added key ERR $\alpha$-targeted genes identified in this study namely DNMT1, DNMT3A, TET2, TET3, AHCY, MAT1A, and $M A T 2 A$, as they were not included in the original dataset (Supplementary Table 1). Unsupervised hierarchical clustering successfully partitioned the tumor profiles into 2 groups distinguished by having either low or high ERR $\alpha$ activity across three independent cohorts obtained from Gene Omnibus and ArrayExpress (GSE2034, GSE24450 and E-TABM-158), thus confirming the validity of the ERR $\alpha$ signature (Fig. 3). Next, we tested for a significant association between the expression of our genes of interest and ERR $\alpha$ activity. Of the genes examined, only DNMT1 transcript levels showed a consistent and significant correlation with $\mathrm{BC}$ tumors bearing high $\mathrm{ERR} \alpha$ activity across the three independent patient cohorts (Fig. 3 and Supplementary Fig. 3). This raises the possibility that simultaneous inhibition of DNMT1 and ERR $\alpha$ activity may offer a therapeutic advantage for the treatment of $\mathrm{BC}$ patients.

\section{Dual inhibition of ERRa and DNMT suppresses BC cell growth in vitro}

While human cancer cells generally harbor global DNA hypomethylation profiles, they also specifically display hypermethylation of promoters of tumor suppressor genes leading to their silencing [26]. Accordingly, inhibition of DNMTs correlates with reduced tumorigenicity often related to re-expression of tumor suppressors [27]. Recently, the FDA-approved DNMT inhibitor 5-azadC (decitabine) was proven effective in treating TNBC [28]. Given our identification of an association between ERR $\alpha$ activity and DNMT1 expression in BC patients, we tested whether ERR $\alpha$ inhibition could further sensitize BC cells to 5azadC. Unexpectedly, 5-azadC alone robustly reduced ERR $\alpha$ protein levels, an effect also observed following the specific knockdown of DNMT1 by RNA interference in 
A

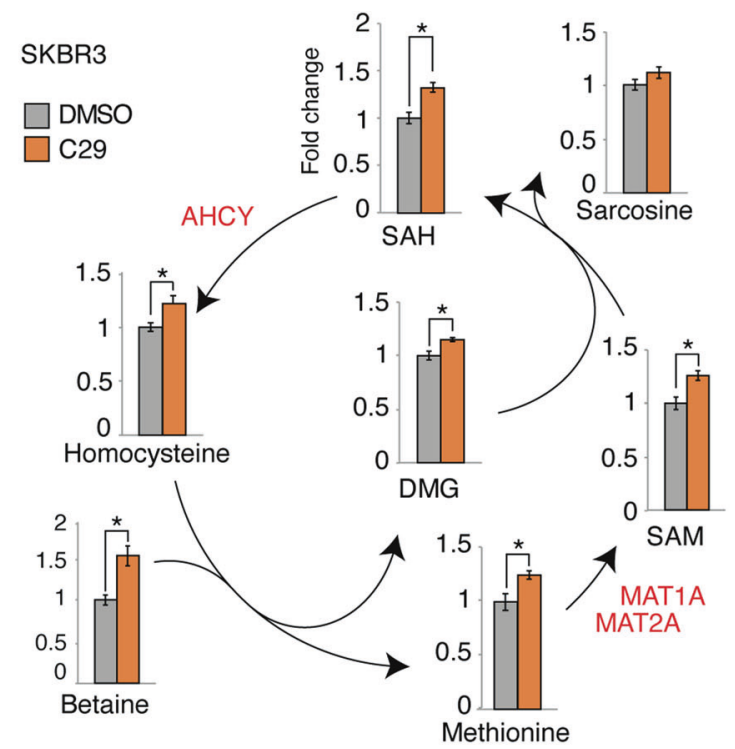

D
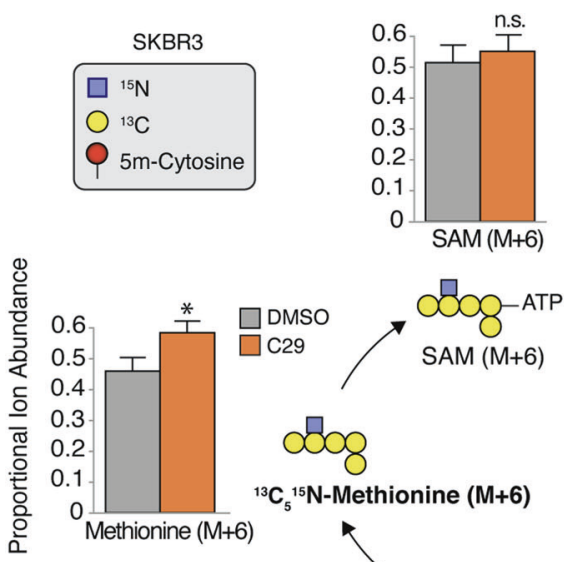

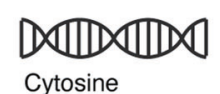
(1)

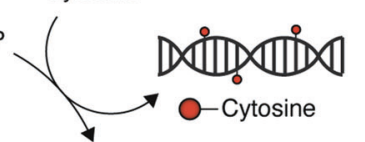

$\$ 008$

${ }^{13} \mathrm{C}_{5}{ }^{15} \mathrm{~N}$-Methionine $(\mathrm{M}+6)$

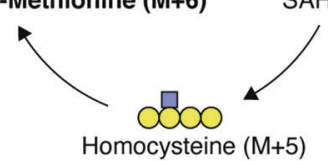

ODOO-ATP

$\mathrm{SAH}(\mathrm{M}+5)$

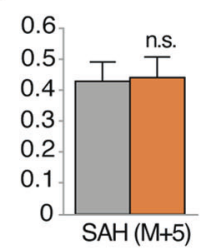

B

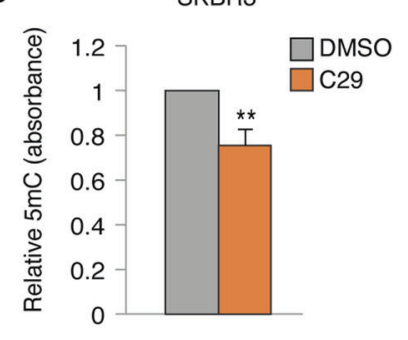

C

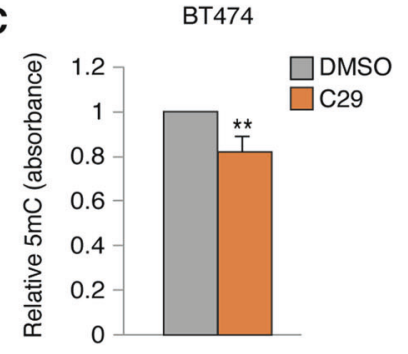

E

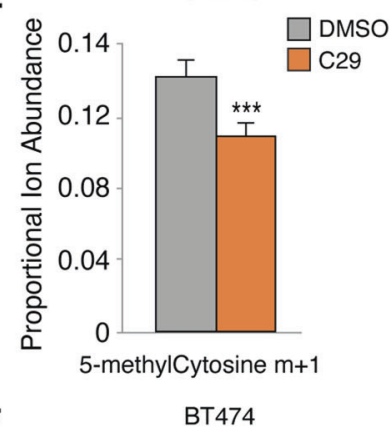

$\mathbf{F}$

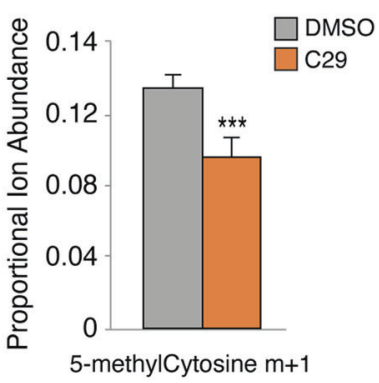

G

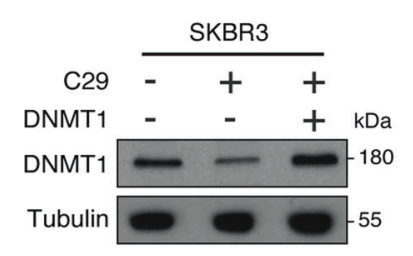

H

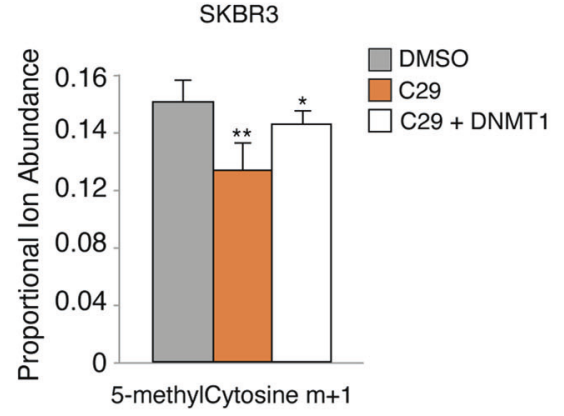

both SKBR3 and BT474 cells (Fig. 4a, b). Further analysis determined that the DNA-demethylating agent 5-azadC induces BC cell autophagy, as indicated by increased levels of the autophagy marker LC3B-II (Fig. 4c), and that blockade of autophagy with bafilomycin A is sufficient to rescue 5-azadC-induced ERR $\alpha$ degradation (Fig. 4d). 5 -azadC has also been shown to promote autophagy in ovarian cancer cells [29]. 
Fig. 2 ERRo is a driver of DNA methylation. a Relative level of metabolites of the methionine cycle upon treatment with C29 for $24 \mathrm{~h}$ in SKBR3 cells. Results reflect three independent experiments each performed with five replicates. b, c Relative 5-methylcytosine levels in SKBR3 (b) and BT474 (c) cells after treatment with C29 for $24 \mathrm{~h}$. Results reflect three independent experiments performed in triplicate. d Stable isotope tracing diagram for ${ }^{13} \mathrm{C}_{5}{ }^{15} \mathrm{~N}$-methionine through the methionine cycle and DNA methylation. Levels of measured labeled methionine cycle intermediates in SKBR3 cells reflect three independent experiments each performed with five replicates. e, f Relative quantification of DNA labeled 5-methylcystosine $(\mathrm{m}+1)$ after treatment with $\mathrm{C} 29$ in the presence of ${ }^{13} \mathrm{C}_{5}{ }^{15} \mathrm{~N}$-methionine for $24 \mathrm{~h}$ in SKBR3 (e) and BT474 (f) cells. Results reflect three independent experiments each performed with five replicates. $\mathbf{g}$ DNMT1 protein expression in SKBR3 cells after treatment with C29 for $24 \mathrm{~h} \pm$ ectopic expression of DNMT1. Tubulin is shown as a loading control. h Relative quantification of DNA labeled 5-methylcystosine $(m+1)$ in SKBR3 cells after treatment with C29 \pm ectopic expression of DNMT1 in the presence of ${ }^{13} \mathrm{C}_{5}{ }^{15} \mathrm{~N}$-methionine for $24 \mathrm{~h}$. Results reflect three independent experiments each performed with five replicates. Data shown in $\mathbf{a}-\mathbf{f}$ and $\mathbf{h}$ represent means \pm SEM. $* p<0.05, * * p<0.01$, $* * * p<0.001$; Student's $t$ test.
Next, we examined the effect of co-treatment with the $\mathrm{ERR} \alpha$ inhibitor $\mathrm{C} 29$ and 5-azadC on BC cellular proliferation. While 5-azadC alone significantly diminished the growth of both the HER2 human SKBR3 and mouse NIC5231 cell lines, C29 amplified the neoplastic effects of the DNMT inhibitor (Fig. 4e, f). Similar beneficial anti-tumor effects of combined ERR $\alpha$ and DNMT inhibitors were observed in the ER+ BC cell line MCF7 and TNBC cell lines MDA-MB-231, MDA-MB-436, and MDA-MB-468 (Fig. $4 \mathrm{~g}-\mathrm{j}$ and Supplementary Fig. $4 \mathrm{a}-\mathrm{d}$ ).

Since ERR $\alpha$ activity is regulated by growth factors and HER2 [30], we wondered whether classical anti-HER2 therapy would also sensitize SKBR3 cells to the DNMT inhibitor 5-azadC. Similar to ERR $\alpha$ loss-of-function in BC cells (Fig. 1e-j), SKBR3 cells treated with lapatinib, a dual epidermal growth factor receptor (EGFR)/human EGFR-2 (HER2) tyrosine kinase inhibitor approved for patients with
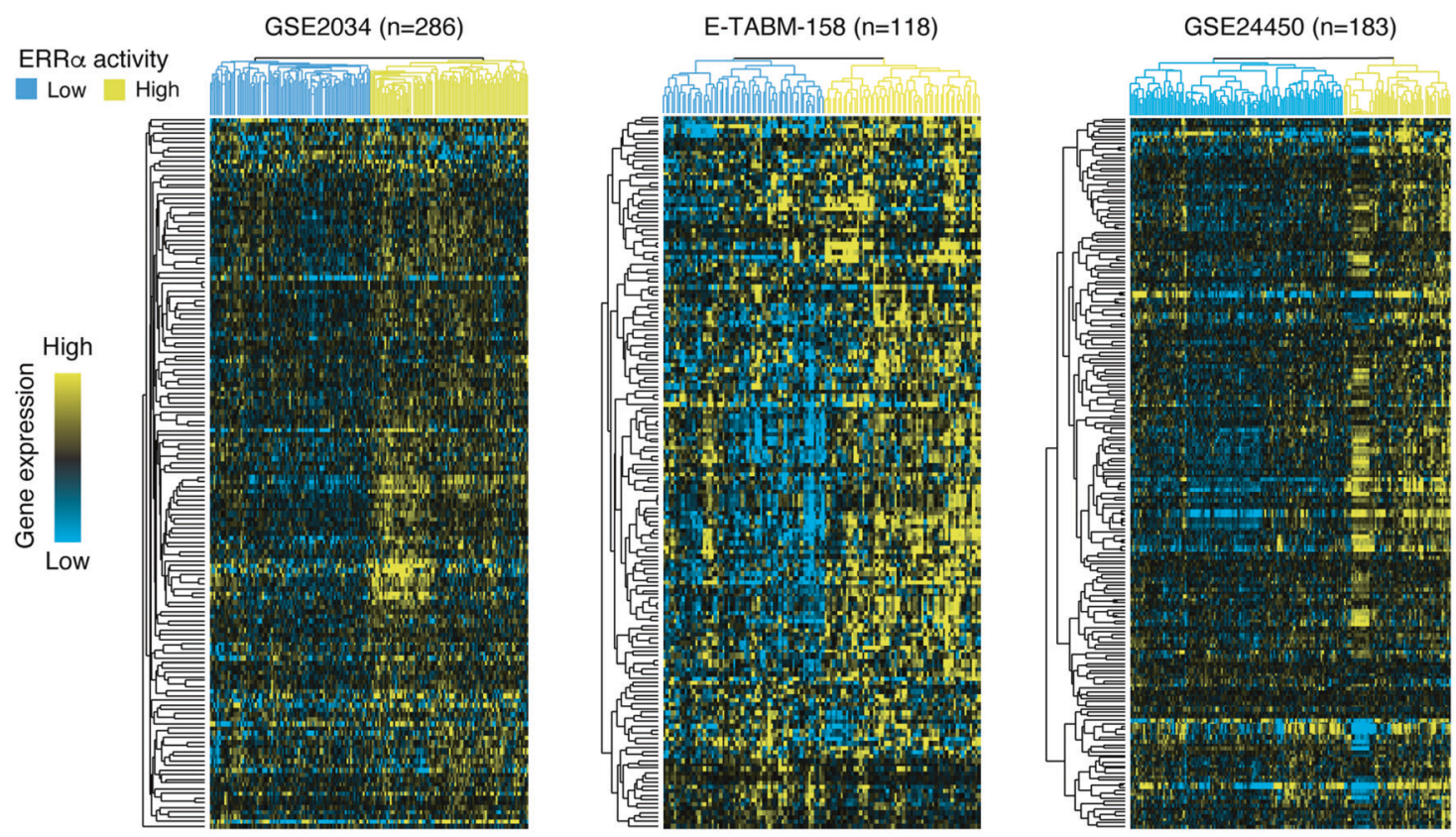
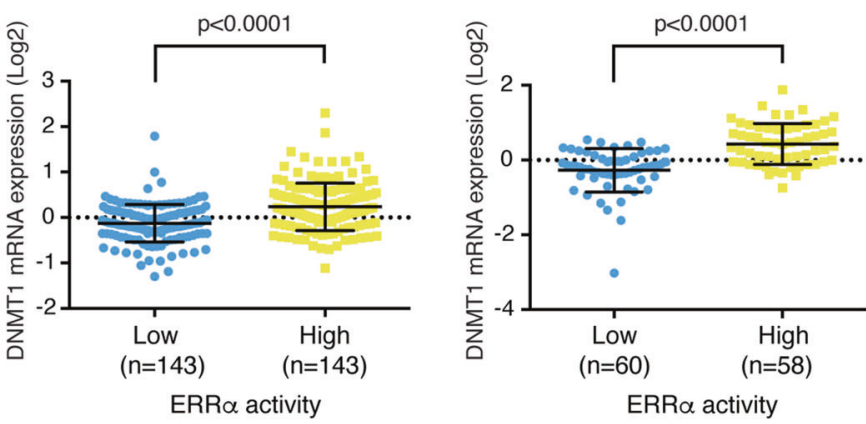

$\mathrm{ERR} \alpha$ activity

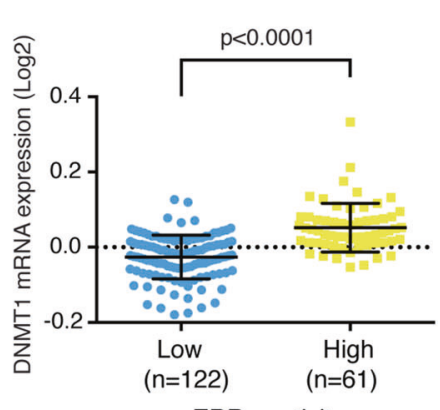

ERR $\alpha$ activity
Fig. 3 ERR $\alpha$ activity positively correlates with DNMT1 expression. Top, unsupervised hierarchical clustering analysis with an ERR $\alpha$-targeted gene signature in three independent BC clinical cohorts. Subtype colors discriminate between patients with low ERR $\alpha$ activity (blue) and patients with high ERR $\alpha$ activity (yellow). Bottom, relative DNMT1 mRNA levels between patients with low and high ERR $\alpha$ activity for each BC cohort. DNMT1 expression values were extracted from microarray data after normalization and were $\log 2$ transformed. Data show means \pm SEM; Student's $t$ test. 
Fig. 4 Dual inhibition of ERR $\alpha$ and DNMT augments $B C$ cell growth hindrance in vitro. a Immunoblots of ERR $\alpha$ and DNMT1 after DNMT1 inhibition by treatment with the DNMT inhibitor 5 -azadC $(5 \mu \mathrm{M})$ for $24 \mathrm{~h}$ in both SKBR3 and BT474 cells.

Tubulin is shown as a loading control. b Immunoblots of ERR $\alpha$ and DNMT1 posttransfection with siRNAs against DNMT1 for $48 \mathrm{~h}$ in both SKBR3 and BT474 cells. Tubulin is shown as a loading control. c Immunoblot of ERRo and the marker of autophagy LC3B in SKBR3 and BT474 cells after treatment with $5 \mu \mathrm{M}$ 5-azadC for $24 \mathrm{~h}$. d Protein levels of ERR $\alpha$ and LC3B in SKBR3 cells after treatment with $5 \mu \mathrm{M} 5$-azadC for $24 \mathrm{~h}$ in combination with $20 \mathrm{nM}$ of the autophagy inhibitor Bafilomycin A1. Bafilomycin A1 was added $6 \mathrm{~h}$ prior harvesting cells. e-j Normalized cell index curves representing proliferation of human HER2+ SKBR3 (e), mouse HER2+ NIC-5231 (f), human ER+ MCF7 (g), TNBC MDA-MB-231 (h), TNBC MDA-MB-436 (i), and TNBC MDA-MB-468 (j) cells in the presence of $\mathrm{C} 29(5 \mu \mathrm{M})$ and/or 5 -azadC $(3 \mu \mathrm{M})$. Three independent experiments each with five replicates were performed and one representative experiment is shown. Data shown in $\mathbf{e}-\mathbf{j}$ represent means \pm SEM. $* * p<0.01, * * * p<0.001$; Student's $t$ test.
A

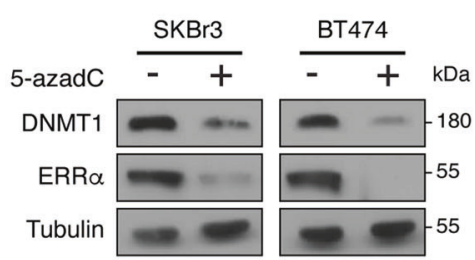

C

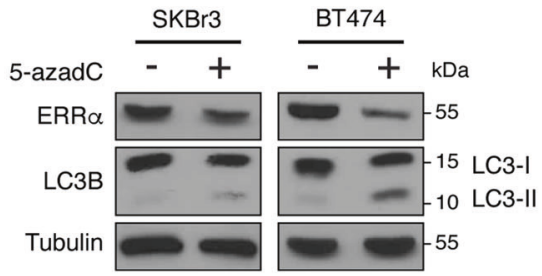

E

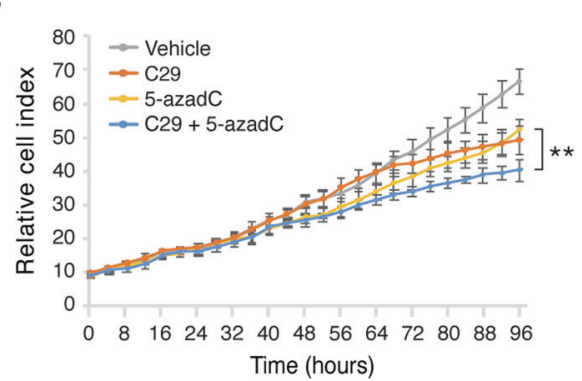

G

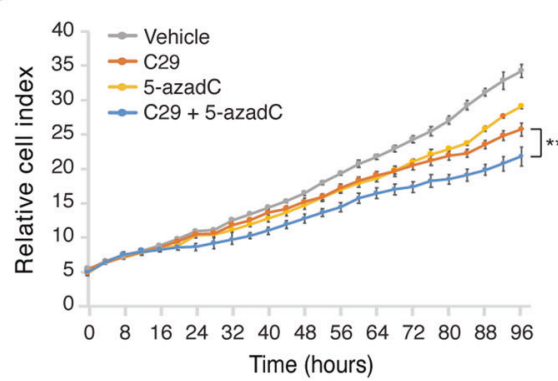

I

MDA-MB-436

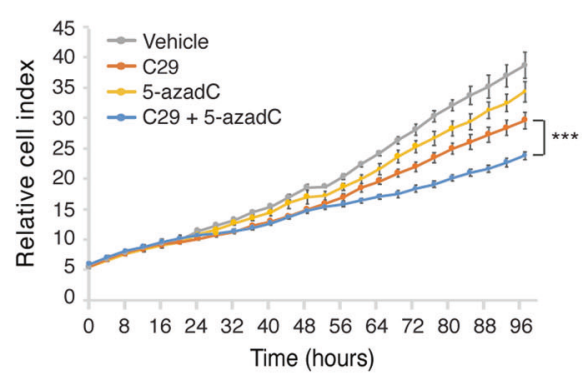

B

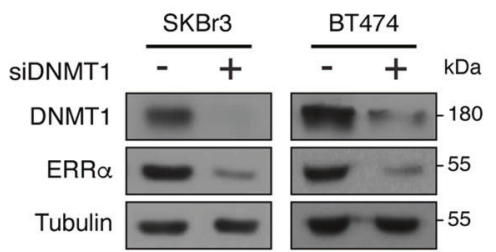

D

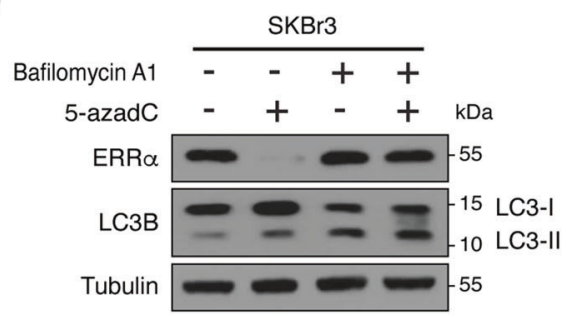

$\mathbf{F}$

NIC-5231

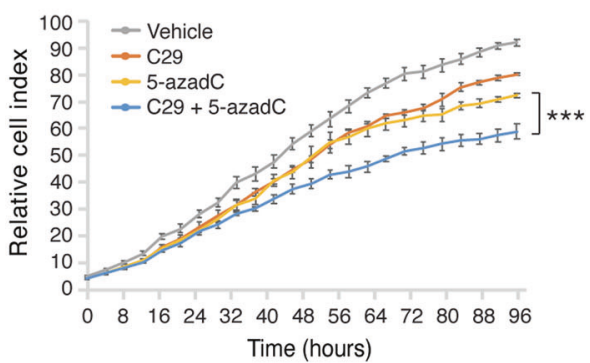

H

MDA-MB-231

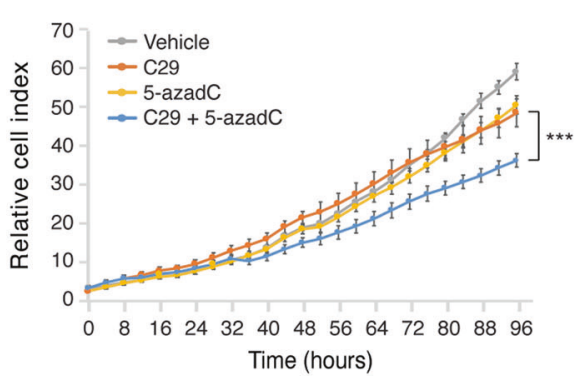

J

MDA-MB-468

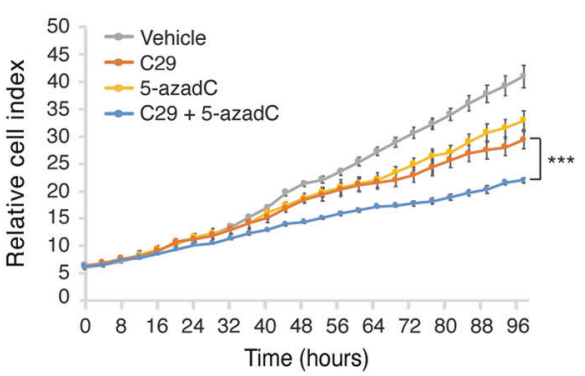

HER2-amplified breast tumors, decreased both ERR $\alpha$ and DNMT1 protein levels (Supplementary Fig. 4e). However, lapatinib was also found to reduce DNMT3a levels (Supplementary Fig. 4e), while ERR $\alpha$ inhibition seemingly stabilized it (Fig. 1e-h, k). Co-treatment of SKBR3 cells with lapatinib and 5-azadC did not further increase the antitumoral effects of each drug alone (Supplementary Fig. 4f), suggesting that the effects of anti-HER2 therapy beyond ERR $\alpha$ inhibition impede the benefit of co-targeting ERR $\alpha$ and DNMT simultaneously. 


\section{C29 enhances the efficacy of 5-azadC on impeding $B C$ tumor development in vivo}

To determine the efficacy of the therapeutic agent 5-azadC in combination with $\mathrm{C} 29$ at suppressing BC tumor growth in vivo, compared to 5 -azadC therapy alone, we used a preclinical mouse cell line-derived xenograft (CDX) model. NIC-5231 cells were injected into the mammary fat pad of NSG mice, and primary tumors were treated with either C29 $(10 \mathrm{mg} / \mathrm{kg})$ and/or 5 -azadC $(1 \mathrm{mg} / \mathrm{kg})$, or vehicle control (Fig. 5a). As anticipated, treatment with the DNAdemethylating drug 5-azadC alone significantly attenuated tumor growth (Fig. 5b, c). Importantly, as observed in vitro, C29 potentiated the tumor suppressive effect of 5-azadC in vivo, thus validating the utility of combining ERR $\alpha$ and DNA methyltransferase inhibitors in the pharmacological intervention of $\mathrm{BC}$ (Fig. 5b, c). Immunoblot analysis of end-point tumors confirmed the positive molecular link between ERR $\alpha$ and DNMT1 expression whereby ERR $\alpha$ loss of function by C29 decreased DNMT1 protein and treatment with 5-azadC reduced ERR $\alpha$ levels (Fig. 5d). Surprisingly, while C29 and 5-azadC independently decreased intra-tumoral levels of 5-methylcytosine, there was no further significant decline in global DNA methylation upon co-treatment compared to the individual drug regimens (Fig. 5e). We therefore hypothesized that C29 and 5 -azadC may have differential effects on promoter-specific DNA methylation that may be otherwise masked by the evaluation of global DNA methylation states. To address this, we performed reduced representation bisulfite sequencing (RRBS) on tumor DNA from these mice. RRBS is a high-throughput technique that offers a large-scale highresolution mapping of DNA methylation across the genome that enriches for regions with high $\mathrm{CpG}$ content such as promoters and repeated sequences [31]. Overall, lower methylated $\mathrm{CpGs}$ were found in all three drug treatment groups compared to control, and the repartitions of the differentially methylated CpGs between introns, exons, promoters and intergenic regions were almost identical across treatment groups (Supplementary Fig. 5a-f). Pathway enrichment analysis of the genes with differentially methylated regions (DMRs) showed no major differences between treatment groups (Supplementary Fig. $5 \mathrm{~g}-\mathrm{i}$ ). We next devised a pipeline as outlined in Fig. $5 f$ to focus our attention on more precise features of the tumor RRBS datasets using several filtering criteria. First, since cancer cells possess specific promoter hypermethylation of tumor suppressor genes, we restricted our analysis to DMRs located within promoter regions (Supplementary Table 2). Given that promoters are under the control of TFs, we looked specifically for TFs targeting these regions and for which $\mathrm{C} 29$ and 5-azadC co-treatment induced promoter hypomethylation. Promoter methylation status was analyzed in silico using the SMARTapp [32], which allowed us to identify TFs possessing hypermethylated $\mathrm{CpG}$ sites that correlate with bad prognosis in $\mathrm{BC}$ patients. Those presenting hypomethylated $\mathrm{CpG}$ sites following the combinatorial drug regimen were retained, given that they offer a therapeutic benefit by allowing for re-expression of tumor suppressor genes. Third, to identify candidate TFs regulating these genes, Homer (Hypergeometric Optimization of Motif EnRichment) software was used for analysis of TF motif enrichment on gene promoters harboring DMRs [33]. In our analysis, 890 genes with promoter DMRs were found uniquely modified in tumors co-treated with $\mathrm{C} 29$ and 5azadC - this included 51 TFs based on a recent article referencing all known human TFs (Fig. 5g and Supplementary Table 3) [34]. Among these $51 \mathrm{TFs}, 9$ were characterized in the SMARTapp as having promoter $\mathrm{CpG}$ hypermethylation and a significant association with bad prognosis in BC. Dual inhibition of ERR $\alpha$ and DNMT specifically induced promoter hypomethylation of six of these nine prognostic TFs: IRF4, FOXE1, VDR, TEAD2, POU3F3, and ZBTB20 (Fig. 5g and Supplementary Table 3). Both VDR and ZBTB20 were previously shown to operate as tumor suppressors in cancer [35, 36]. IRF4 has also been found to have both oncogenic and tumor suppressive activities in hematological cancer [37, 38]. Further examination with TF motif enrichment analysis revealed strong differences in potential TFs implicated in the response to each treatment (Fig. 5h). Intriguingly, the IRF4 motif was specifically enriched after co-treatment with C29 and 5-azadC (Fig. 5h), a condition found to demethylate the IRF4 promoter (Fig. $5 \mathrm{~g}$ ). Thus, the computational analysis highlights IRF4 as a potential active participant in mediating the anti-tumor effects observed. Alongside this implication, we noted that the strongest motif enriched in the combined treatment group was for ETV1, a member of the ETS family of TFs and well-known as an oncogene in several cancer types including BC [39]. Considering that a role for IRF4 in BC has never been explored, we next sought to investigate its potential tumor suppressor activity in this context.

\section{Combined C29 and 5-azadC treatment reverses IRF4 promoter hypermethylation in BC}

We first aimed to confirm in silico the methylation status of IRF4 in cancer. The SMARTapp revealed the existence of $16 \mathrm{CpGs}$ with available methylation data for this gene. Aggregating the mean methylation levels of all CpGs, IRF4 is significantly hypermethylated in $\mathrm{BC}$ as well as in almost every other cancer type within the TCGA collection (Fig. 6a and Supplementary Fig. 6a). Specifically, 10 out of 16 CpGs are localized in the IRF4 promoter (Fig. 6b). According to SMARTapp, these ten sites are all 
A

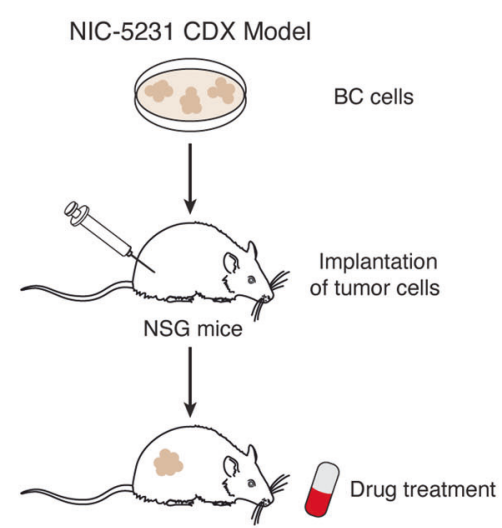

D
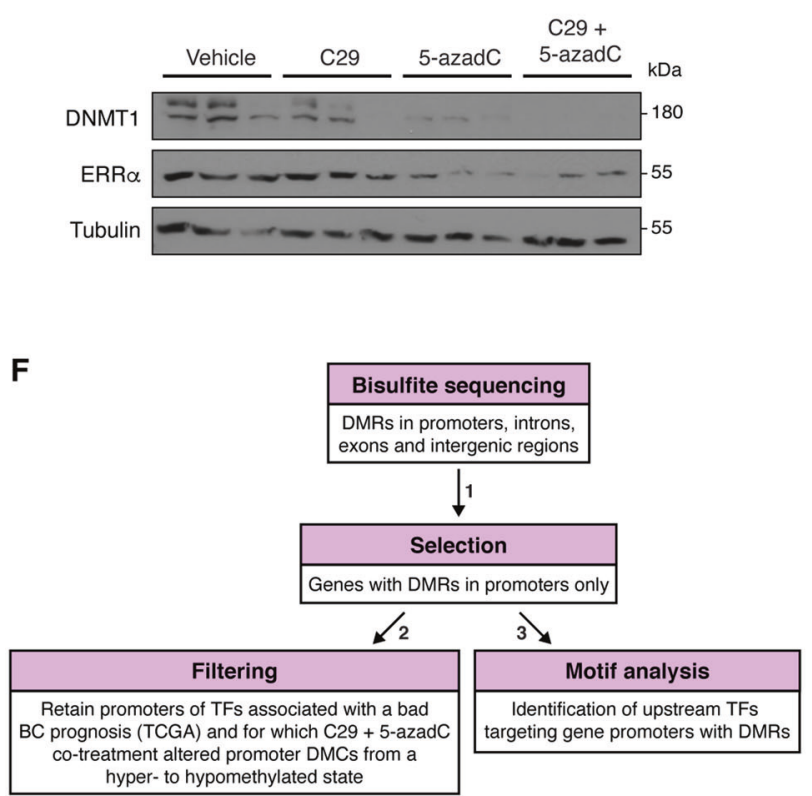

B

C

E
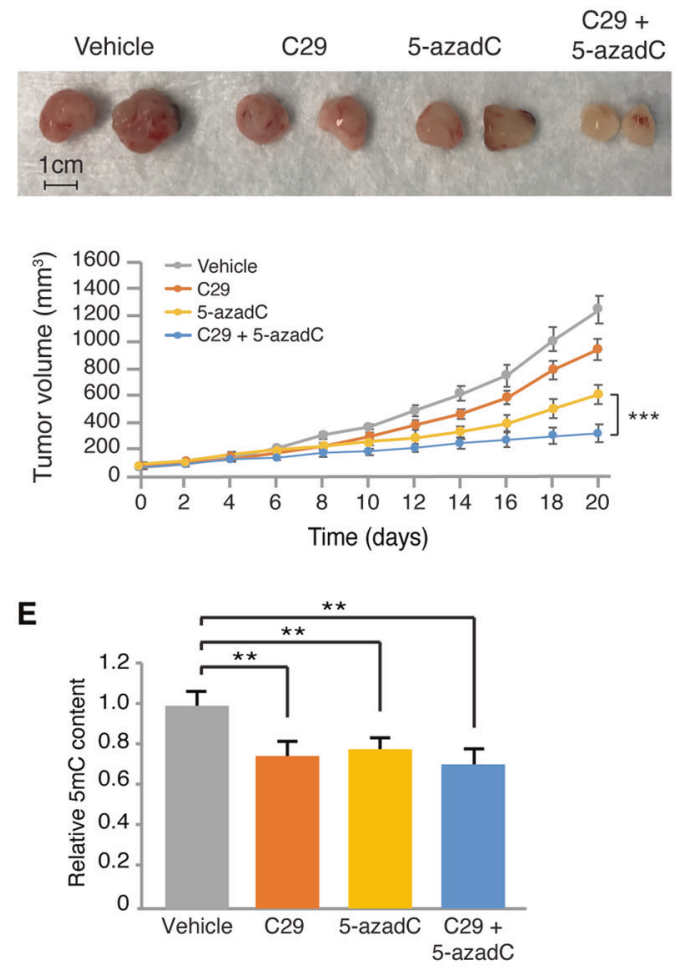

G

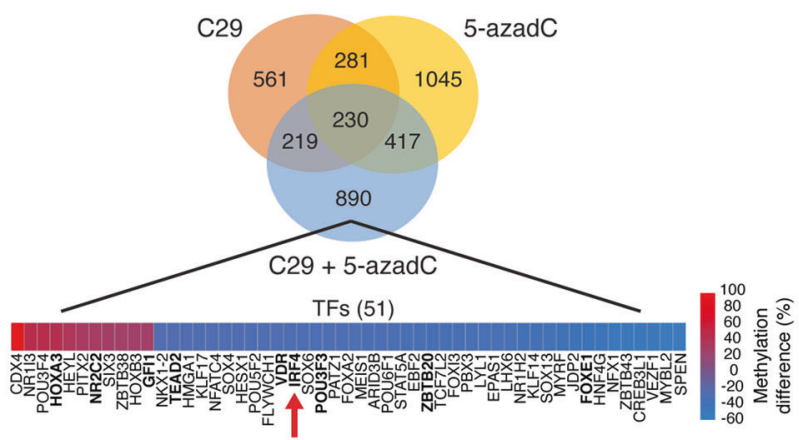

H Subset of TF enriched motifs in promoters with DMRs modulated by the drug treatments

\begin{tabular}{ccc}
\multicolumn{3}{c}{ C29 } \\
\hline TF & Motif & p-value \\
\hline spdef & AATCCGGA & $1 e-186$ \\
Arid5a & ATCCCTAATATC & $1 e-70$ \\
Nr2f2 & ATTCTGAGCCGG & $1 e-56$ \\
TR4 & AGCTCATAGGGC & $1 e-55$ \\
ZNF416 & ACCTGGGCACGT & $1 e-53$ \\
Nkx2-2 & CATGTCAAGTGA & $1 e-51$ \\
Sp100 & ACCGACCATT & $1 e-51$ \\
TEAD2 & ATATCCAGGTC & $1 e-46$
\end{tabular}

\begin{tabular}{ccc}
\hline \multicolumn{3}{c}{ 5-azadC } \\
\hline TF & Motif & p-value \\
\hline Elk1 & ACTECCGG & $1 \mathrm{e}-616$ \\
Sp100 & GCCGGGTAAT & $1 \mathrm{e}-84$ \\
IRF6 & CAATCTCCGGC & $1 \mathrm{e}-55$ \\
PRox1 & AGACGTCTCCGG & $1 \mathrm{e}-45$ \\
Glis2 & GGGCGGCCGCGA & $1 \mathrm{e}-45$ \\
Ebf & CCTCTCTTCGCG & $1 \mathrm{e}-42$ \\
Zfp281 & TCTCCCCCAATC & $1 \mathrm{e}-39$ \\
MzF1 & GTTAGTTGGGGA & $1 \mathrm{e}-39$
\end{tabular}

\begin{tabular}{ccc}
\multicolumn{3}{c}{ C29 + 5-azadC } \\
\hline TF & Motif & p-value \\
\hline Etv1 & TTCACCGG & $1 \mathrm{e}-788$ \\
Etv2 & GACCGGCAATAT & $1 \mathrm{e}-64$ \\
Nr2f2 & ATTCTGACCCGG & $1 \mathrm{e}-61$ \\
TBX5 & AACACCTCGCTA & $1 \mathrm{e}-54$ \\
DUX4 & GGTTAGGTTAA & $1 \mathrm{e}-54$ \\
CUX1 & CATTGATAATT & $1 \mathrm{e}-50$ \\
SP1 & ACGCACCTCCCC & $1 \mathrm{e}-49$ \\
\hline IRF4 & CCGTTTCA & $1 \mathrm{e}-43$ \\
\hline
\end{tabular}

hypermethylated in $\mathrm{BC}$ with nine displaying a significant positive correlation with poor overall survival (Fig. 6b-e and Supplementary Fig. 6b). Moreover, their methylation status negatively correlated with gene-level expression, confirming that hypermethylation of the IRF4 promoter would lead to IRF4 silencing (Fig. 6f-h and Supplementary Fig. $6 \mathrm{c}$ ). To validate this, we selected one $\mathrm{CpG}$ site within each of the three $\mathrm{CpG}$ islands found within the IRF4 promoter (Fig. 6b), and performed methylation-specific quantitative PCR (MS-qPCR), a technique that allows direct 
Fig. 5 Decitabine and $\mathrm{C29}$ act in concert to suppress $\mathrm{BC}$ tumor growth in vivo. a Schematic illustrating the establishment of a mouse BC CDX model from NIC-5231 cells for pharmacological drug testing in a pre-clinical setting. $\mathbf{b}$ Representative images of tumors extracted at endpoint (20 days post-treatment) from mouse mammary fat pads illustrating the effect of the different drug regimens on tumor size. c Tumor growth curves following administration of C29 $(10 \mathrm{mg} / \mathrm{kg})$, 5 -azadC $(1 \mathrm{mg} / \mathrm{kg})$ or a combination of both drugs $(n=5$ for each group). d Immunoblots of DNMT1 and ERR $\alpha$ in tumors after a 20-day drug regimen ( $n=3$ samples per group are shown). Tubulin is shown as a loading control. e Relative quantification of 5-methylcytosine levels in tumors after 20 days of treatment ( $n=5$ per group). f Computational pipeline developed for the identification of genes of interest from the RRBS analysis on tumors of mice treated with the different drug regimens. $g$ Venn diagram representing the overlap of the number of DMRs in gene promoters after treatment with C29, 5 -azadC or a combination of both drugs. A heatmap of the 51 transcription factors found with differential promoter methylation after C29 and 5-azadC co-treatment is shown. Red represents promoter hypermethylation, while blue designates promoter hypomethylation. The red arrow points at the transcription factor IRF4. $\mathbf{h}$ Subset of TF motif enrichment analysis performed on the total list of DMRs identified following treatment with $\mathrm{C} 29,5$-azadC or a combination of both drugs. The IRF4 motif (boxed in red) was specifically enriched in promoters with DMRs of tumors co-treated with C29 + 5-azadC. Data shown in (c) and (e) represent means \pm SEM. $* * p<0.01, * * * p<$ 0.001; Student's $t$ test.

evaluation of the methylation status of a specific $\mathrm{CpG}$ site [40]. Compared to control SKBR3 cells treated with vehicle, the level of methylation at all three $\mathrm{CpG}$ sites tested was significantly reduced by the addition of either $\mathrm{C} 29$ or 5-azadC. Remarkably, these effects were additive, as cotreatment of the drugs effectively abrogated IRF4 promoter CpG methylation (Fig. 6i-k).

\section{The suppressive effects of concomitant ERRa and DMNT inhibition on BC growth is dependent on IRF4 derepression}

To verify IRF4 expression, we performed RT-PCR on SKBR3 cells treated with $\mathrm{C} 29$ and/or 5-azadC, or vehicle control, using primers designed to amplify a $200 \mathrm{bp}$ cDNA region of IRF4. In accordance with the state of IRF4 promoter methylation (Fig. 6i-k), we could not detect IRF4 in SKBR3 cells under basal conditions or in response to C29 or 5-azadC treatment alone (Fig. 7a). However, coadministration of both drugs resulted in amplification of the IRF4 cDNA (Fig. 7a), thus confirming that concurrent inhibition of ERR $\alpha$ and DMNT can successfully derepress the IRF4 gene that was silenced by promoter hypermethylation. We next sought to provide evidence for a tumor suppressive action of IRF4 re-activation underlying the therapeutic benefit to a combined ERR $\alpha$ and DNMT drug therapy in BC. Accordingly, SKBR3 cells were first infected with either a non-specific shRNA (shNTC) or with one of two different shRNAs targeting IRF4 (Fig. 7b).
Subsequently, the cells were exposed to either a combined $\mathrm{C} 29$ and 5-azadC drug regimen or vehicle control and the impact on cellular growth was evaluated (Fig. $7 \mathrm{c}$ and Supplementary Fig. 7). As expected, knockdown of IRF4 had no impact on cell growth in the vehicle condition, a context in which IRF4 is already silenced (Supplementary Fig. 7). In stark contrast, while $\mathrm{C} 29$ and 5-azadC inhibited cell proliferation, this effect was demonstrated to be IRF4dependent as shRNA-mediated suppression of IRF4 could significantly restore cell growth capabilities, underscoring an anti-proliferative function of IRF4 in BC cells (Fig. 7c). Finally, we interrogated the PRECOG website (https:// precog.stanford.edu/) [41] to investigate the correlation between IRF4 expression and patient overall survival in BC. Our analysis confirmed a positive correlation between IRF4 expression and favorable patient outcome, thus validating our findings that IRF4 plays a tumor suppressor role in BC (Fig. 7d, e).

\section{Discussion}

In this study, we identified a new ERR $\alpha$-dependent regulatory pathway conserved across species linking cell metabolism and DNA methylation. We show that ERR $\alpha$, a major regulator of cellular metabolism, coordinates SAM synthesis through the methionine cycle while driving DNMT1 expression to promote DNA methylation. Genetic or pharmacological inhibition of ERR $\alpha$ repressed DNMT1 expression, the activity of the methionine cycle and, ultimately, global DNA methylation. Reciprocally, we uncovered that inhibiting DNMT1 diminishes ERR $\alpha$ levels, suggesting that DNMT1 directly influences cell metabolism. ERR $\alpha$ activity and DNMT1 expression were found to positively correlate in $\mathrm{BC}$ patients independent of $\mathrm{BC}$ subtype, reinforcing the molecular link between these two genomic regulators. Of clinical relevance, targeting ERR $\alpha$ with the specific inhibitor C29 significantly increased the sensitivity of BC cells to the DNMT inhibitor decitabine both in vitro and in vivo. A large-scale analysis of DNA methylation further revealed that co-treatment with both drugs alters promoter methylation of a specific set of genes, leading to the identification and functional characterization of IRF4, found to possess tumor suppressor activity in BC and derepressed in this context.

While DNA methylation is tightly bound to the metabolic state of the cell, active DNA demethylation also occurs and depends on the availability of specific metabolites. Indeed, we observed that ERR $\alpha$ inhibition induced the expression of the demethylase TET3, suggesting that ERR $\alpha$ could also be involved in active DNA demethylation (Fig. 1). However, we speculate that loss of DNA methylation linked to ERR $\alpha$ inhibition is more likely due to a lack 


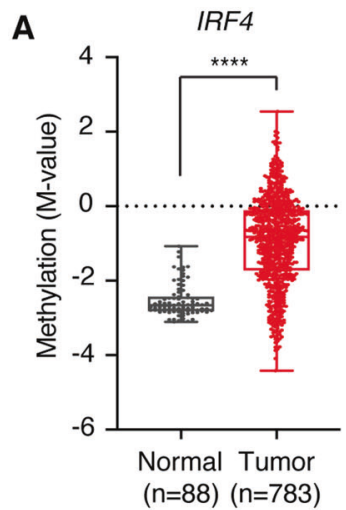

C

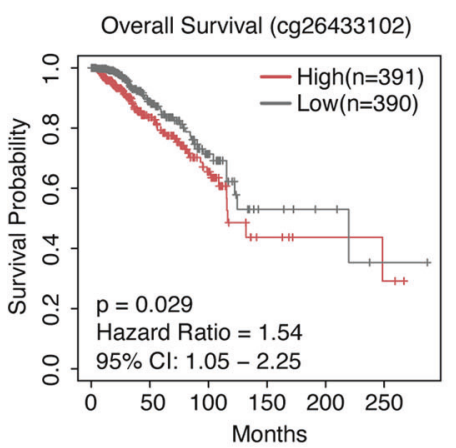

$\mathbf{F}$

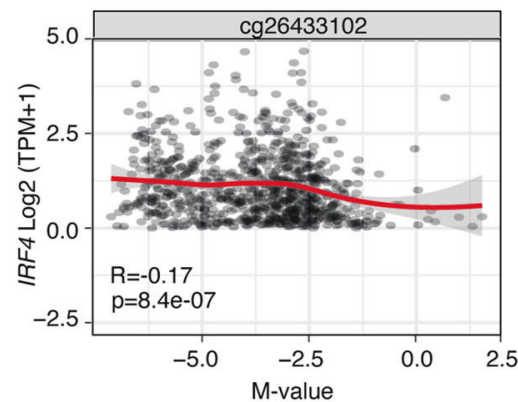

I

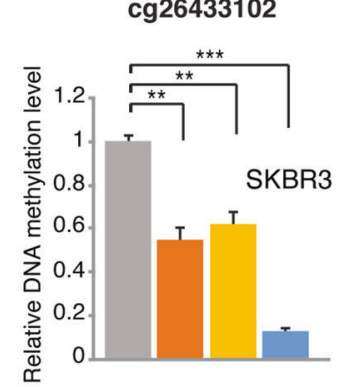

B
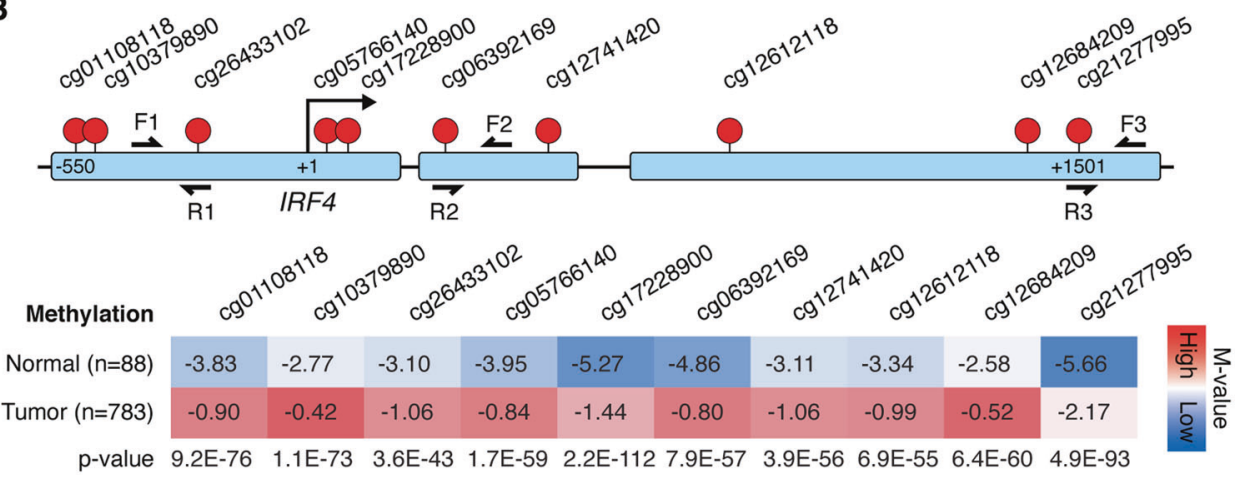

D

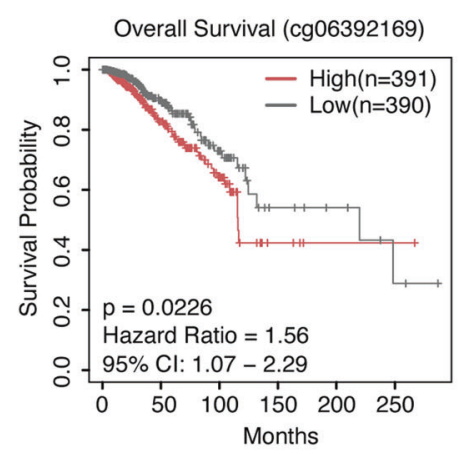

E

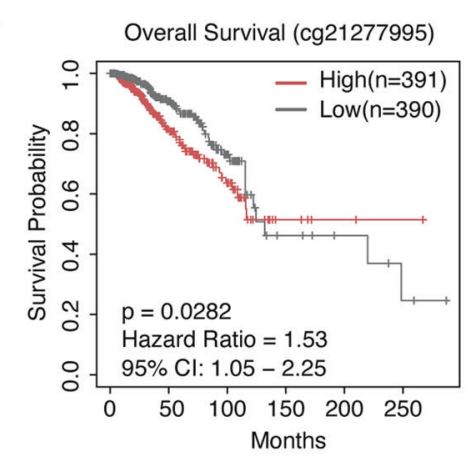

G

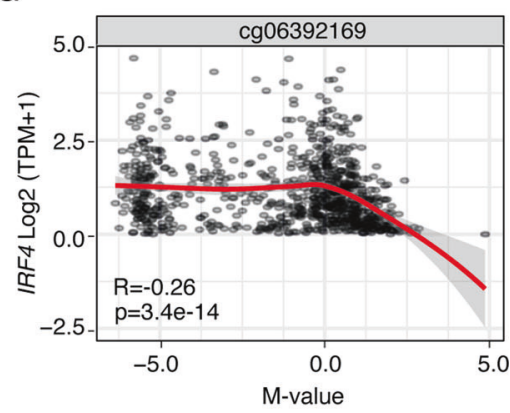

H

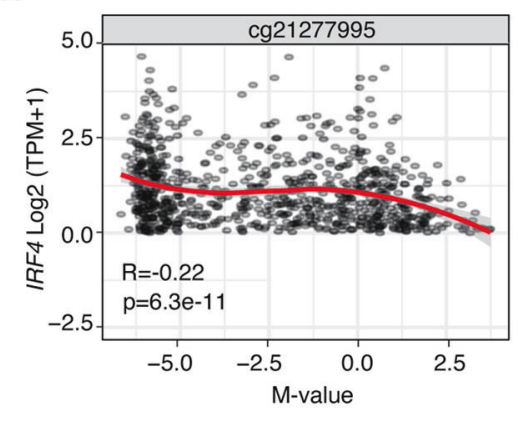

K

$\operatorname{cg} 21277995$
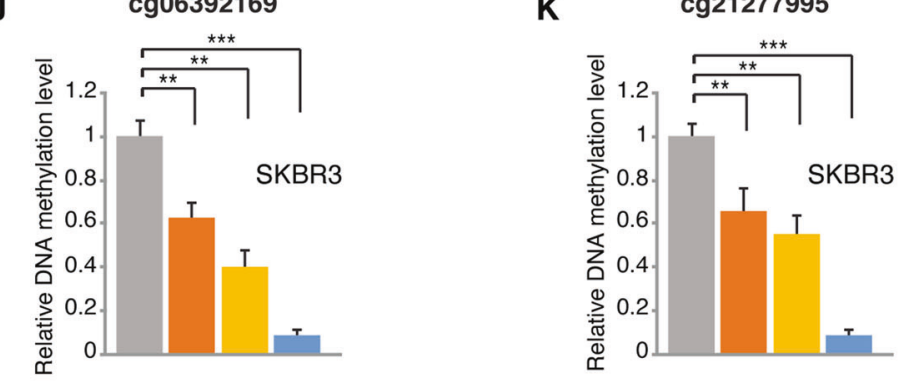

Ionso

C29 5-azadC $\quad$ C29 + 5-azadC

of DNMT1 activity than to an increase in demethylase activity. This hypothesis is supported by our recent report demonstrating that ERR $\alpha$ inhibition depletes the available pool of $\alpha \mathrm{KG}$ [21], a required cofactor of dioxygenase enzymes such as the TET DNA demethylases. $\alpha K G$ is mostly synthesized through glutaminolysis in cancer, a pathway regulated by ERR $\alpha$ in $\mathrm{BC}$ [42], and is an important intermediate of the TCA cycle involved in many cellular functions such as anti-oxidation, protein, and lipid synthesis, as well as cellular respiration [43].

The methionine cycle is central to several essential metabolic pathways. Notably, methionine combines with 
Fig. 6 The IRF4 promoter is hypermethylated in $\mathrm{BC}$ patients and correlates with poor overall survival. a Box plot showing the methylation status of IRF4 in BC patients using the mean aggregation of all $16 \mathrm{CpGs}$ referenced within the SMARTapp obtained from the TCGA project. The outer limits of the box represent the 25th (lower quartile) and 75th percentile (upper quartile) with the median value shown inside. Whiskers extend to the lowest and highest values. $* * * * p<0.0001$; Student's $t$ test. b Schematic representation of the IRF4 promoter containing ten $\mathrm{CpG}$ sites identified by SMARTapp found within three $\mathrm{CpG}$ islands (blue bars). The corresponding relative mean methylation levels for each of the 10 promoter-specific $\mathrm{CpG}$ sites in normal tissue and $\mathrm{BC}$ tumor samples ( $M$-value) are shown. The associated $p$ values were calculated using the Student's $t$ test. The pairs of primers used for MS-qPCR analysis in $\mathbf{i}-\mathbf{k}$ are represented as F1-R1 for cg26433102, F2-R2 for cg06392169, and F3-R3 for cg21277995. c-e Kaplan-Meier survival curves derived from the SMARTapp illustrating the correlation between the methylation status ( $M$-value) of the IRF4-associated CpG sites cg26433102 (c), cg06392169 (d), and cg21277995 (e) with BC patient overall survival. f-h Spearman correlation curves obtained from the SMARTapp showing the association between the methylation status ( $M$-value) of the IRF4-associated CpG sites cg26433102 (f), cg06392169 (g), and cg21277995 (h) and IRF4 gene-level expression in BC patients $(n=853)$. i-k Relative methylation levels of the IRF4-associated CpG sites cg26433102 (i), $\operatorname{cg} 06392169(\mathbf{j})$, and $\operatorname{cg} 21277995(\mathbf{k})$ after treatment with C29 $(5 \mu \mathrm{M})$, 5 -azadC $(3 \mu \mathrm{M})$ or a combination of both drugs for 7 days in SKBR3 cells. Data presented in (i-k) show means \pm SEM. $* * p<0.01, * * * p<$ 0.001 ; Student's $t$ test.

ATP to produce SAM, the principal methyl donor for methylation of proteins, DNA, RNA, and lipids [44]. By donating one carbon for methylation, SAM generates SAH, the precursor of homocysteine which can ultimately produce glutathione, the main cellular antioxidant [45]. However, regeneration of methionine from homocysteine has been proven to be low in cancer cells, rendering them highly dependent on exogenous methionine [14, 46]. Hence, dietary methionine restriction is under intense investigation as a potential anti-cancer therapy and has shown promising results in improving metabolism, increasing lifespan and preventing cancer cell growth in numerous contexts [47-52]. Given the clear importance of methionine in cancer, the mechanisms through which methionine cycle enzymes are regulated must be well understood. Here, we show that ERR $\alpha$ positively regulates the expression of several methionine cycle genes, influencing the levels of intermediate metabolites. These findings are significant in the light of our recent work showing that $E R R \alpha$ represses the folate cycle and that ERR $\alpha$ inhibition leads to an increase in purine biosynthesis [18]. Considering the intimate relationship between these two pathways as the recycling of methionine links the methionine and the folate cycles, nucleotide synthesis and NADH/NADPH production, it raises the question as to why ERR $\alpha$ would regulate these programs differently [53-55]. One possible reason stems from a recent study demonstrating that the methionine and folate cycles compete for metabolites involved in DNA methylation, nucleotide synthesis, and anti-oxidation [56].
A

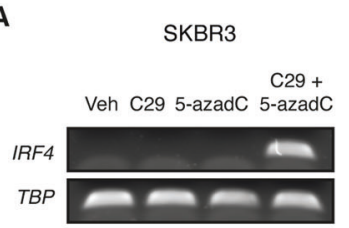

B $\quad$ SKBR3 (C29 +5-azadC)

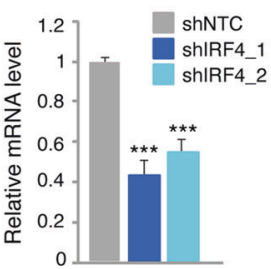

C

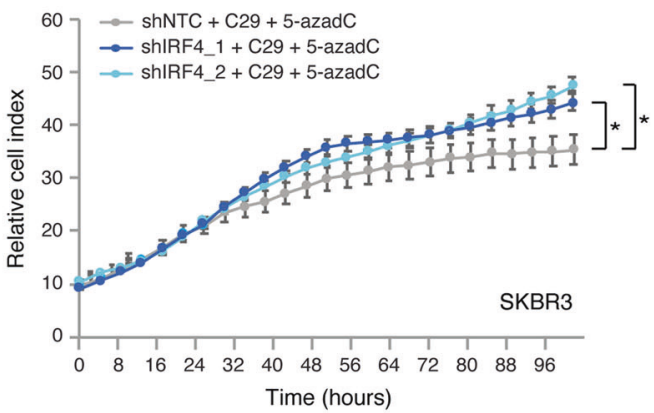

D

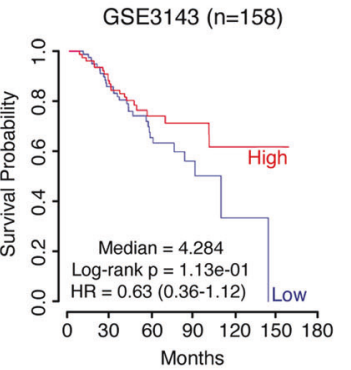

E $\operatorname{GSE7390~(n=155)}$
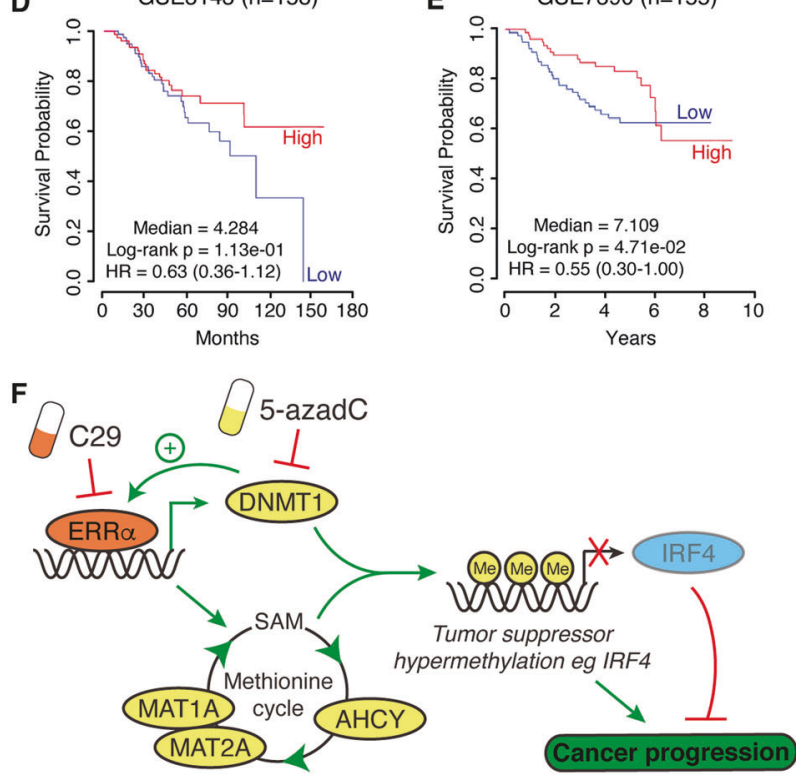

This suggests that ERR $\alpha$ might act as a switch or sensor to balance these processes [57], which could be particularly important in cancer cells where high nucleotide synthesis, elevated oxidative states, and DNA hypomethylation are often observed.

Cancer cell DNA is characterized by promoter hypermethylation of tumor suppressor genes that induces their silencing. Thus, demethylating agents such as 5-azadC have been proposed as anti-cancer therapies with the intention of re-establishing tumor suppressor expression. 5-azadC is currently used clinically for the treatment of myelodysplastic syndrome and other leukemias, where the drug has received FDA approval [58]. However, clinical development of this drug is still prohibited in solid tumors due to 
Fig. 7 IRF4 is a tumor suppressor in BC re-expressed upon cotreatment with $\mathbf{C 2 9}$ and 5-azadC. a Agarose gel electrophoresis showing PCR product amplification of IRF4 cDNA following treatment with $\mathrm{C} 29(5 \mu \mathrm{M}), 5$-azadC $(3 \mu \mathrm{M})$ or a combination of both drugs for 7 days in SKBR3 cells. b Relative mRNA levels of IRF4 in SKBR3 cells infected with shRNAs against IFR4 after treatment with C29 $(5 \mu \mathrm{M}), 5$-azadC $(3 \mu \mathrm{M})$ or a combination of both drugs for 7 days. c Normalized cell index curves representing proliferation of SKBR3 cells infected with either a control shRNA (shNTC) or two different shRNAs against IRF4 in the presence of C29 $(5 \mu \mathrm{M})$ and 5 -azadC $(3 \mu \mathrm{M})$. Data represent one experiment performed with five replicates. d, e Kaplan-Meier survival curves representing the positive correlation between IRF4 mRNA expression and overall survival of $\mathrm{BC}$ patients in two independent cohorts consisting of 158 patients (GSE3143) (d) and 155 patients (GSE7390) (e). f Model illustrating how pharmacological inhibition of the inter-connected factors ERR $\alpha$ and DNMT1 can halt BC progression by simultaneously repressing methionine cycle metabolism and DNA methylation. Consequent epigenetic modulation impinges on the newly attributed tumor suppressor gene IRF4 in BC with $\mathrm{C} 29$ and 5-azadC co-treatment promoting IRF4 derepression. Data shown in $\mathbf{b}$ and $\mathbf{c}$ represent means \pm SEM. ${ }^{*} p<0.05, * * * p<0.001$; Student's $t$ test.

substantial toxicity [59]. Nevertheless, anti-tumor activity of 5 -azadC has been reported in BC patients with a response rate of up to $50 \%$ and, more recently, levels of DNMT proteins have been proposed as biomarkers for decitabine response in TN BC $[28,60]$. These observations suggest that a better understanding of the mechanisms of regulation of DNA methylation in BC might help to improve the use of demethylating agents in anti-cancer regimens. Here, our work shows DNMT1 supporting ERR $\alpha$ as a driver of DNA methylation to fuel $\mathrm{BC}$ development, thus highlighting a therapeutic advantage of targeting both factors (Fig. 7f). We have shown that the combinatorial drug therapy induces a new anti-tumor mechanism involving promoter demethylation of $I R F 4$, a previously unknown tumor suppressor gene in this context. In support of our findings, it is interesting to note that significantly higher methylation in IRF4 was previously observed in HER2 + breast tumors in relation to normal breast tissues [61], and that high IRF4 expression associates with improved outcome in HER2+ node-negative BC [62]. While our work shows that IRF4 silencing indeed promotes tumor growth, further studies will be needed to fully decipher the exact anti-tumor program driven by IRF4 in BC, as well as the signaling pathways controlling its expression in both normal and $\mathrm{BC}$ tissues.

Taken together, our study offers a new therapeutic avenue for BC treatment by simultaneously targeting the methionine cycle and DNA methylation via the combined actions of ERR $\alpha$ and DNMT inhibitors, while potentially reducing the toxic side-effects associated to high doses of demethylating agents. Finally, it will be interesting to establish whether this combination therapeutic approach could be beneficial for other non-hematopoietic cancers.

\section{Materials and methods}

Details of all methods are found in Supplementary Information.

\section{Accession numbers}

RRBS datasets from mouse tumor xenografts have been deposited in the NCBI Gene Expression Omnibus (GEO; https://ncbi.nlm.nih.gov/geo/) under the accession number GSE149603.

Acknowledgements We acknowledge contributions from the Metabolomics Core Facility (MCF) of the GCRC, as well as technical assistance from Dr. Daina Avizonis, Mariana De Sa Tavares Russo, Gaëlle Bridon, and Luc Choinière. We thank all members of the V.G. laboratory for discussions, Xiaojing Li for technical assistance and Dr. David J. Papadopoli for guidance with metabolic studies. This work was supported by a Terry Fox Research Institute Program Project Team Grant on Oncometabolism (VG and JS-P), a Foundation Grant from the Canadian Institutes of Health Research (CIHR) to VG, and an operating grant from CIHR to JS-P (MOP-106603). MV was a recipient of a post-doctoral fellowship from CIHR. SM was supported by a Vanier Canada Graduate Scholarship-CIHR. EAW was a recipient of a post-doctoral fellowship from CIHR.

Author contributions Conception and design: MV, VG. Development of methodology: MV, SM, CRD, JS-P. Acquisition of data: MV, SW, EA-W. Data analysis and interpretation: MV, SM, CRD, JS-P, VG. Writing of the manuscript: MV, CRD, VG. Study supervision: VG

\section{Compliance with ethical standards}

Conflict of interest The authors declare that they have no conflict of interest.

Publisher's note Springer Nature remains neutral with regard to jurisdictional claims in published maps and institutional affiliations.

Open Access This article is licensed under a Creative Commons Attribution 4.0 International License, which permits use, sharing, adaptation, distribution and reproduction in any medium or format, as long as you give appropriate credit to the original author(s) and the source, provide a link to the Creative Commons license, and indicate if changes were made. The images or other third party material in this article are included in the article's Creative Commons license, unless indicated otherwise in a credit line to the material. If material is not included in the article's Creative Commons license and your intended use is not permitted by statutory regulation or exceeds the permitted use, you will need to obtain permission directly from the copyright holder. To view a copy of this license, visit http://creativecommons. org/licenses/by/4.0/.

\section{References}

1. Razin A, Cedar H. DNA methylation and gene expression. Microbiol Rev. 1991;55:451-8.

2. Luo C, Hajkova P, Ecker JR. Dynamic DNA methylation: In the right place at the right time. Science. 2018;361:1336-40.

3. Smith ZD, Meissner A. DNA methylation: roles in mammalian development. Nat Rev Genet. 2013;14:204-20. 
4. Farlik M, Halbritter F, Muller F, Choudry FA, Ebert P, Klughammer J, et al. DNA methylation dynamics of human hematopoietic stem cell differentiation. Cell Stem Cell. 2016;19:808-22.

5. Putiri EL, Robertson KD. Epigenetic mechanisms and genome stability. Clin Epigenetics. 2011;2:299-314.

6. Nilsson E, Jansson PA, Perfilyev A, Volkov P, Pedersen M, Svensson MK, et al. Altered DNA methylation and differential expression of genes influencing metabolism and inflammation in adipose tissue from subjects with type 2 diabetes. Diabetes. 2014;63:2962-76.

7. Castellano-Castillo D, Moreno-Indias I, Sanchez-Alcoholado L, Ramos-Molina B, Alcaide-Torres J, Morcillo S, et al. Altered adipose tissue DNA methylation status in metabolic syndrome: relationships between global DNA methylation and specific methylation at adipogenic, lipid metabolism and inflammatory candidate genes and metabolic variables. J Clin Med. 2019;8:87.

8. Sanchez-Mut JV, Heyn H, Vidal E, Moran S, Sayols S, DelgadoMorales R, et al. Human DNA methylomes of neurodegenerative diseases show common epigenomic patterns. Transl Psychiatry. 2016;6:e718.

9. Hermann A, Goyal R, Jeltsch A. The Dnmt1 DNA-(cytosine-C5)methyltransferase methylates DNA processively with high preference for hemimethylated target sites. J Biol Chem. 2004;279:48350-9.

10. Martin EM, Fry RC. Environmental influences on the epigenome: exposure-associated DNA methylation in human populations. Annu Rev Public Health. 2018;39:309-33.

11. Okano M, Bell DW, Haber DA, Li E. DNA methyltransferases Dnmt3a and Dnmt3b are essential for de novo methylation and mammalian development. Cell. 1999;99:247-57.

12. Tahiliani M, Koh KP, Shen Y, Pastor WA, Bandukwala H, Brudno $\mathrm{Y}$, et al. Conversion of 5-methylcytosine to 5hydroxymethylcytosine in mammalian DNA by MLL partner TET1. Science. 2009;324:930-5.

13. Ito S, D'Alessio AC, Taranova OV, Hong K, Sowers LC, Zhang Y. Role of Tet proteins in $5 \mathrm{mC}$ to $5 \mathrm{hmC}$ conversion, ES-cell selfrenewal and inner cell mass specification. Nature. 2010;466: 1129-33.

14. Maddocks OD, Labuschagne CF, Adams PD, Vousden KH. Serine metabolism supports the methionine cycle and DNA/RNA methylation through de novo ATP synthesis in cancer cells. Mol Cell. 2016;61:210-21.

15. Deblois G, Giguère V. Oestrogen-related receptors in breast cancer: control of cellular metabolism and beyond. Nat Rev Cancer. 2013;13:27-36.

16. Giguère V. Transcriptional control of energy homeostasis by the estrogen-related receptors. Endocr Rev. 2008;29:677-96.

17. Audet-Walsh E, Giguère V. The multiple universes of estrogenrelated receptor $\alpha$ and $\gamma$ in metabolic control and related diseases. Acta Pharmacol Sin. 2015;36:51-61.

18. Audet-Walsh E, Papadopoli DJ, Gravel SP, Yee T, Bridon G, Caron $\mathrm{M}$, et al. The PGC- $1 \alpha / \mathrm{ERR} \alpha$ axis represses one-carbon metabolism and promotes sensitivity to anti-folate therapy in breast cancer. Cell Rep. 2016;14:920-31.

19. Park S, Safi R, Liu X, Baldi R, Liu W, Liu J. et al. Inhibition of ERRalpha prevents mitochondrial pyruvate uptake exposing NADPH-generating pathways as targetable vulnerabilities in breast cancer. Cell Rep. 2019;27:3587-601.e3584.

20. Ariazi EA, Clark GM, Mertz JE. Estrogen-related receptor $\alpha$ and estrogen-related receptor $\gamma$ associate with unfavorable and favorable biomarkers, respectively, in human breast cancer. Cancer Res. 2002;62:6510-8.

21. Deblois G, Smith HW, Tam IS, Gravel SP, Caron M, Savage P, et al. ERR $\alpha$ mediates metabolic adaptations driving lapatinib resistance in breast cancer. Nat Commun. 2016;7:12156.
22. Deblois G, Hall JA, Perry MC, Laganière J, Ghahremani M, Park $\mathrm{M}$, et al. Genome-wide identification of direct target genes implicates estrogen-related receptor $\alpha$ as a determinant of breast cancer heterogeneity. Cancer Res. 2009;69:6149-57.

23. Chang CY, Kazmin D, Jasper JS, Kunder R, Zuercher WJ, McDonnell DP. The metabolic regulator ERR $\alpha$, a downstream target of HER2/IGF-1R, as a therapeutic target in breast cancer. Cancer Cell. 2011;20:500-10.

24. Patch RJ, Searle LL, Kim AJ, De D, Zhu X, Askari HB, et al. Identification of diaryl ether-based ligands for estrogen-related receptor $\alpha$ as potential antidiabetic agents. J Med Chem. 2011;54:788-808.

25. Schade B, Rao T, Dourdin N, Lesurf R, Hallett M, Cardiff RD, et al. PTEN deficiency in a luminal ErbB-2 mouse model results in dramatic acceleration of mammary tumorigenesis and metastasis. $\mathrm{J}$ Biol Chem. 2009;284:19018-26.

26. Esteller M. Epigenetics in cancer. N. Engl J Med. 2008;358: 1148-59.

27. Phan NL, Trinh NV, Pham PV. Low concentrations of 5-aza-2'deoxycytidine induce breast cancer stem cell differentiation by triggering tumor suppressor gene expression. Onco Targets Ther. 2016;9:49-59.

28. Yu J, Qin B, Moyer AM, Nowsheen S, Liu T, Qin S, et al. DNA methyltransferase expression in triple-negative breast cancer predicts sensitivity to decitabine. J Clin Investig. 2018;128:2376-88.

29. Chen MY, Liao WS, Lu Z, Bornmann WG, Hennessey V, Washington MN, et al. Decitabine and suberoylanilide hydroxamic acid (SAHA) inhibit growth of ovarian cancer cell lines and xenografts while inducing expression of imprinted tumor suppressor genes, apoptosis, G2/M arrest, and autophagy. Cancer. 2011;117:4424-38.

30. Barry JB, Giguère V. Epidermal growth factor-induced signaling in breast cancer cells results in selective target gene activation by orphan nuclear receptor estrogen-related receptor $\alpha$. Cancer Res. 2005;65:6120-9.

31. Meissner A, Gnirke A, Bell GW, Ramsahoye B, Lander ES, Jaenisch R. Reduced representation bisulfite sequencing for comparative high-resolution DNA methylation analysis. Nucleic Acids Res. 2005;33:5868-77.

32. Li Y, Ge D, Lu C. The SMART App: an interactive web application for comprehensive DNA methylation analysis and visualization. Epigenetics Chromatin. 2019;12:71.

33. Heinz S, Benner C, Spann N, Bertolino E, Lin YC, Laslo P, et al. Simple combinations of lineage-determining transcription factors prime cis-regulatory elements required for macrophage and B cell identities. Mol Cell. 2010;38:576-89.

34. Lambert SA, Jolma A, Campitelli LF, Das PK, Yin Y, Albu M, et al. The human transcription factors. Cell. 2018;175:598-9.

35. Huss L, Butt ST, Borgquist S, Elebro K, Sandsveden M, Rosendahl A, et al. Vitamin D receptor expression in invasive breast tumors and breast cancer survival. Breast Cancer Res. 2019;21:84.

36. de la Rosa J, Weber J, Friedrich MJ, Li Y, Rad L, Ponstingl H, et al. A single-copy Sleeping Beauty transposon mutagenesis screen identifies new PTEN-cooperating tumor suppressor genes. Nat Genet. 2017;49:730-41.

37. Acquaviva J, Chen X, Ren R. IRF-4 functions as a tumor suppressor in early B-cell development. Blood. 2008;112:3798-806.

38. Shaffer AL, Emre NC, Romesser PB, Staudt LM. IRF4: immunity. malignancy! therapy? Clin Cancer Res. 2009;15:2954-61.

39. Oh S, Shin S, Janknecht R. ETV1, 4 and 5: an oncogenic subfamily of ETS transcription factors. Biochim Biophys Acta. 2012;1826:1-12.

40. Herman JG, Graff JR, Myohanen S, Nelkin BD, Baylin SB. Methylation-specific PCR: a novel PCR assay for methylation status of $\mathrm{CpG}$ islands. Proc Natl Acad Sci USA. 1996;93: 9821-6. 
41. Gentles AJ, Newman AM, Liu CL, Bratman SV, Feng W, Kim D, et al. The prognostic landscape of genes and infiltrating immune cells across human cancers. Nat Med. 2015;21:938-45.

42. McGuirk S, Gravel S-P, Deblois G, Papadopoli D, Faubert B, Wegner $\mathrm{A}$, et al. PGC-1 $\alpha$ supports glutamine metabolism in brreast cancer cells. Cancer Metab. 2013;1:22.

43. Zdzisinska B, Zurek A, Kandefer-Szerszen M. Alphaketoglutarate as a molecule with pleiotropic activity: wellknown and novel possibilities of therapeutic use. Arch Immunol Ther Exp. 2017;65:21-36.

44. Bauerle MR, Schwalm EL, Booker SJ. Mechanistic diversity of radical S-adenosylmethionine (SAM)-dependent methylation. J Biol Chem. 2015;290:3995-4002.

45. Lu SC. Glutathione synthesis. Biochim Biophys Acta. 2013;1830:3143-53.

46. Mehrmohamadi M, Liu X, Shestov AA, Locasale JW. Characterization of the usage of the serine metabolic network in human cancer. Cell Rep. 2014;9:1507-19.

47. Jeon H, Kim JH, Lee E, Jang YJ, Son JE, Kwon JY, et al. Methionine deprivation suppresses triple-negative breast cancer metastasis in vitro and in vivo. Oncotarget. 2016;7:67223-34.

48. Komninou D, Leutzinger Y, Reddy BS, Richie JP Jr. Methionine restriction inhibits colon carcinogenesis. Nutr Cancer. 2006;54: 202-8.

49. Barcena C, Quiros PM, Durand S, Mayoral P, Rodriguez F, Caravia XM, et al. Methionine restriction extends lifespan in progeroid mice and alters lipid and bile acid metabolism. Cell Rep. 2018;24:2392-403.

50. Lee BC, Kaya A, Ma S, Kim G, Gerashchenko MV, Yim SH, et al. Methionine restriction extends lifespan of Drosophila melanogaster under conditions of low amino-acid status. Nat Commun. 2014;5:3592.

51. Gao X, Sanderson SM, Dai Z, Reid MA, Cooper DE, Lu M, et al. Dietary methionine influences therapy in mouse cancer models and alters human metabolism. Nature. 2019;572: $397-401$.
52. Sanderson SM, Gao X, Dai Z, Locasale JW. Methionine metabolism in health and cancer: a nexus of diet and precision medicine. Nat Rev Cancer. 2019;19:625-37.

53. Shlomi T, Fan J, Tang B, Kruger WD, Rabinowitz JD. Quantitation of cellular metabolic fluxes of methionine. Anal Chem. 2014;86:1583-91.

54. Labuschagne CF, van den Broek NJ, Mackay GM, Vousden KH, Maddocks OD. Serine, but not glycine, supports one-carbon metabolism and proliferation of cancer cells. Cell Rep. 2014;7:1248-58.

55. Fan J, Ye J, Kamphorst JJ, Shlomi T, Thompson CB, Rabinowitz JD. Quantitative flux analysis reveals folate-dependent NADPH production. Nature. 2014;510:298-302.

56. Cao S, Zhu X, Zhang C, Qian H, Schuttler HB, Gong J, et al. Competition between DNA methylation, nucleotide synthesis, and antioxidation in cancer versus normal tissues. Cancer Res. 2017;77:4185-95.

57. Vernier M, Dufour CR, McGuirk S, Scholtes C, Li X, Bourmeau $\mathrm{G}$, et al. Estrogen-related receptors are targetable ROS sensors. Genes Dev. 2020;34:544-59.

58. Issa JP. Optimizing therapy with methylation inhibitors in myelodysplastic syndromes: dose, duration, and patient selection. Nat Clin Pract Oncol. 2005;2:S24-29.

59. Momparler RL, Bouffard DY, Momparler LF, Dionne J, Belanger K, Ayoub J. Pilot phase I-II study on 5-aza-2'-deoxycytidine (Decitabine) in patients with metastatic lung cancer. Anticancer Drugs. 1997;8:358-68.

60. Cowan LA, Talwar S, Yang AS. Will DNA methylation inhibitors work in solid tumors? A review of the clinical experience with azacitidine and decitabine in solid tumors. Epigenomics. 2010;2:71-86.

61. Lindqvist BM, Wingren S, Motlagh PB, Nilsson TK. Whole genome DNA methylation signature of HER2-positive breast cancer. Epigenetics. 2014;9:1149-62.

62. Heimes AS, Madjar K, Edlund K, Battista MJ, Almstedt K, Gebhard S, et al. Prognostic significance of interferon regulating factor 4 (IRF4) in node-negative breast cancer. J Cancer Res Clin Oncol. 2017;143:1123-31. 